\title{
Impact of Numerics on the Predictive Capabilities of Reacting Flow LES
}

\author{
Peter A. T. Cocks ${ }^{*}$, Marios C. Soteriou ${ }^{\dagger}$ and Vaidyanathan Sankaran ${ }^{\ddagger}$ \\ United Technologies Research Center, East Hartford, CT, 06108, USA
}

\begin{abstract}
Combustors in modern aerospace propulsion systems are highly optimized devices and further enhancements to their performance will require advanced predictive modeling techniques. The LES approach has potential to be such a technique, but demonstration of its true predictive capability remains a challenge. In this paper, a methodical study is performed to assess the impact of numerical error on the predictive capabilities of LES and to distinguish it from error arising from physical modeling. Four codes are employed to simulate the bluff body stabilized flame experiment of Volvo Flygmotor AB. The physical models (governing equations and sub-grid models) are kept identical between the codes so that the physical modeling error is the same. For the same reason, computational grids are also kept identical since the sub-grid models are grid dependent. Results indicate that all codes produce a very similar solution for the non-reacting flow in terms of large scale unsteady vortex shedding behavior, mean profiles and second order turbulence statistics, on a suitably refined grid. This solution is also very close to the experimentally observed behavior of the flow. In contrast, for the reacting case, solutions from the various codes exhibit significant differences when analyzed under the same metrics and with similar or even finer grid resolution than that used for the non-reacting case. These differences are large enough to change the macroscopic behavior of the flow, e.g. some codes predict symmetric vortex shedding while others predict asymmetric shedding and some codes show strong recirculation zones while others show stagnant regions behind the bluff body. It is also shown that none of the solutions truly predicts the experimentally observed flow. Given that the physical sub-grid models and computational grids are the same in all the simulations, the inconsistency between results points to a significant impact of numerical methods and, hence, numerical error. The need for mitigation of this impact is highlighted, along with the need for metrics detailing how LES should be applied to reacting flows. It is suggested that both of these issues should be the focus of future work.
\end{abstract}

Keywords: Large Eddy Simulation; Premixed combustion; Bluff body stabilized flame; Numerical error

\section{Introduction}

Modern aerospace combustors are challenging traditional operability envelopes in pursuit of increased efficiency, with improved stability and reduced emissions. The harsh and complex environments prevalent in such combustors are thrusting modeling and simulation tools to the forefront of design for next generation technology. As a result, there has been a push to develop high fidelity predictive computational methods to facilitate this development. In this vein, Large Eddy Simulation (LES) is increasingly replacing traditional approaches such as Reynolds Averaged Navier Stokes (RANS) in the Computational Fluid Dynamics (CFD) simulation of turbulent reacting flows.

LES attempts to directly capture the large problem-dependent scales of the flow whilst only requiring a model for the smaller, more universal, turbulence structures. This separation of scales is achieved by filtering the governing system of equations. Traditionally, this filtering process is in practice done implicitly by the grid, where scales smaller than the grid spacing ('filter width') are not captured. Since the unsteady interactions of multiple scales are directly captured there is an increased expectation of solution quality and predictive capability from LES, compared to RANS based CFD methods. However, due to the requirement of fine grid resolutions and due to the timedependent nature of the approach, LES is an expensive proposition, especially for industrial scale problems.

\footnotetext{
${ }^{*}$ Research Engineer, cockspa@utrc.utc.com, +1-860-610-7808 (corresponding author)

${ }^{\dagger}$ Research Fellow, SoteriMC@utrc.utc.com

* Research Engineer, SankarV@utrc.utc.com
} 
For reacting flow LES, physical sub-grid models are required which represent both the presence of the sub-grid scale turbulence structures and the interaction of these structures with the chemical processes occurring in combustion. Currently, these physics are modelled separately, and the respective models are termed as the turbulence and combustion sub-grid models. However, despite considerable investment in sub-grid model development, the reacting flow LES approach practiced today is not truly predictive and this has limited its utilization for industrial applications. There are many possible reasons for this lack of predictive capability, including:

i. A lack of computational set-up criteria. Although some criteria have been developed to guide the setup and execution of incompressible non-reacting simuations ${ }^{1}$ (e.g., criteria for grid resolution), such criteria do not exist for compressible reacting flows. A detailed understanding of the interactions between combustion and turbulence, and the additional interaction with numerical error in LES, is required before such criteria can reliably be developed.

ii. The fidelity of combustion sub-grid models. Since high fidelity combustion sub-grid models are computationally expensive, lower fidelity models are often employed which have their origins in RANS modelling. Given the complexity of the physical phenomena that need to be captured by these models their fidelity controls the solution. In addition, the assumptions behind many combustion sub-grid models are often only validated using simple test cases, and may fail under conditions found in realistic devices.

iii. The impact of numerical error. Numerical errors can interact with errors from physical sub-grid models. This is particularly significant given the common practice of using the grid as a filter, thereby allowing numerical and physical sub-grid model errors to be of the same order. The two types of error can interact, adding or partially canceling ${ }^{2}$, increasing or reducing the total error, respectively, and leading to well established grid convergence problems ${ }^{4}$. A not so well established corollary of the error interaction issue is that the performance of physical sub-grid models in different CFD solvers, which use different numerical schemes and hence have different numerical error characteristics, will be inconsistent. A clear demonstration of this problem will be presented this paper.

iv. Inadequate experimental data. Detailed experimental data sets are often obtained at conditions of modest exothermicity, which is not representative of most realistic combustors. This limits the effective assessment of reacting flow LES, since under these conditions the impact of combustion on the flow field is weak and non-reacting flow dynamics dominate. In addition, data sets tend to lack the detail required to comprehensively validate LES models, particularly so in their ability to report unsteady information. Measurement capabilities also have limitations under high pressure conditions and gaining optical access for such experiments can prove challenging.

There has been extensive research aimed at improving the predictive capabilities of reacting flow LES, however it has primarily been focused on addressing item (ii) above. As already implied, physical sub-grid model development without prior assessment of numerical error is likely to be unreliable. Therefore, this paper investigates item (iii); an important step in the assessment of the predictive capabilities of reacting flow LES.

Numerical errors such as truncation (e.g., dissipative and dispersive), aliasing and commutative errors can corrupt a LES solution in a way that it does not a DNS or a RANS solution ${ }^{\S}$, because in LES the grid is commonly used as the simultaneous physical and numerical filter. While the introduction of sub-grid models is meant to mitigate the effect of physical filtering by representing the impact of the unresolved sub-grid scales on the resolved grid, the fact that these sub-grid models are typically linked to the grid size makes the errors from them comparable to the numerical error. This is further complicated by the fact that the physical manifestation of the errors can also be similar, e.g., dissipative truncation error versus error from a dissipative sub-grid model such as the Smagorinsky model. As a result of the above, the ability to separate and mitigate the impacts of numerical and physical sub-grid model errors is very challenging and is typically not pursued. For example, numerical studies presented at the TNF workshop on Sandia flames ${ }^{3}$, as well as other studies ${ }^{5}$, employ different numerical schemes, physical sub-grid models, computational grids and boundary conditions, for the simulation of a given test case. In such efforts it is

$\S$ In both DNS and RANS, numerically generated scales are separated from physical scales. This is achieved by making sure the grid is much finer than the smallest possible physical scale in DNS, and by making sure the grid is much finer than the smallest modelled scale in RANS. 
difficult to judge the impact of numerical methods, versus physical sub-grid modeling, on the solutions obtained. Since the analysis of physical sub-grid model error is often the focus of such efforts, the impact of numerical error is not well appreciated by LES practitioners.

There have been exceptions to the above. Some studies ${ }^{6-14}$ have been conducted to estimate and mitigate the impact of numerical error separately from sub-grid model error in non-reacting LES. These studies are restricted to simplified and canonical configurations and as such do not demonstrate the true impact of this error in realistic problems. Moreover, no such studies exist for LES of reacting flows. Finally, no generic and affordable ${ }^{15}$ approach for mitigation has yet been found and the methods employed to date are complex and expensive and therefore have not made it into mainstream use. Such methods are the error landscape approach ${ }^{16,17}$ and explicit filtering ${ }^{8-13}$.

The main objective of this paper is to focus attention on the significant impact of numerical error on reacting flow LES and to demonstrate the challenges facing physical sub-grid model development and transition, in the presence of this error. The focus is to demonstrate how numerical error can impact the predictive capabilities of reacting-flow LES, but not to analyze why this is happening or to demonstrate how to fix it. These latter analyses are important and natural next steps to the work presented here and should be the subject of future research. To achieve these objectives, a careful and impartial investigation of the impact of numerical methods on reacting flow LES is presented by using four different compressible reacting flow LES codes to simulate the same test case. The codes employed include university-based, open source, and commercially available codes, all of which utilize different numerical schemes for the discretization of the governing equations. In order to enable a one-on-one comparison and in order to isolate the impact of numerical error from these schemes on the solutions obtained, the same physical sub-grid models and computational grids are employed for all the codes investigated.

The bluff-body stabilized premixed flame experiments performed under the Volvo Flygmotor AB program ${ }^{18-20}$ are considered the most suitable choice for the research conducted here. This test case is generic in the sense that it includes many of the physical processes present in practical combustors, such as anchored flames, regions of recirculation and shear layers. Yet, it is simple enough to be relatively inexpensive to simulate. In addition, while not comprehensive, the experimental measurements are extensive and include both velocity and scalar data in terms of mean profiles and second order turbulence statistics, as well as dynamic information in the form of frequency spectra.

The Volvo experiment does suffer from the low heat release problem noted earlier (the burning efficiency of the experiment is only $18 \%$ ). However, due to the specific nature of this test case, even this small heat release is able to cause a substantial impact of combustion on the flow field, and hence this can be used to assess predictive capabilities of reacting LES. Specifically, in this experiment, the well documented Von Karman like shedding behind a bluff body in a non-reacting flow is suppressed by combustion ${ }^{21}$ to instead form relatively symmetric shedding. This is explicitly noted in the Volvo study and is quantified in measurements taken downstream of the bluff-body that show the elimination of the characteristic Von Karman shedding frequency in the reacting flow ${ }^{19}$.

Even though the Volvo experiments have been simulated by modelers worldwide ${ }^{22-33}$, most of these studies $^{24,27,28,31,32,33}$ fail to completely capture the switch from asymmetric to symmetric flame shape. For example, some of the studies report a symmetric flame in the near field but an asymmetric flame away from the bluff-body. It is also interesting to note that most of these studies showed reasonable agreement with experiments in the mean data, in spite of failing to capture this dominant observable switch in the flame shape. This discrepancy highlights the need for experimental data sets to include detailed quantitative measurements of flow-field statistical quantities, as well as qualitative information such as high-speed video. This large variability in results also highlights the limitation in predictive capabilities of reacting flow LES, as employed today.

The paper is organized as follows. Section II is used to describe the experimental set-up and available validation data for the case to be simulated. This is followed by a description of the LES codes, numerical schemes, combustion models, grids and time steps used in this study. Section III is used to report, discuss and analyze the results from the non-reacting and reacting LES simulations. Conclusions are then drawn.

\section{Background}

\section{A. Volvo Test Case and Validation Data}

The Volvo test case consists of a bluff body flame-holder centered in a constant area duct. The upstream air flow is distributed over the inlet cross-section by a critical orifice plate, which acoustically isolates the combustor from the air supply system. For the reacting experiments, gaseous propane is injected through a multi-orifice critical flow 
injector, upstream of the air supply system. Levels of turbulence in the inlet section are controlled through the use of honeycombs or screens, which also ensures a uniform equivalence ratio mixture enters the combustor.

The conditions under consideration in this paper are an air mass flow rate of $0.6 \mathrm{~kg} / \mathrm{s}$, Reynolds number of 48,000 (defined using the bluff body characteristic length) and inlet temperature of $288 \mathrm{~K}$. For the reacting case the equivalence ratio is 0.65 ; this equivalence ratio gives an adiabatic flame temperature of $1784 \mathrm{~K}$. The bluff body cross section is an equilateral triangle with characteristic length, $\mathrm{D}$, equal to $40 \mathrm{~mm}$. The height and depth of the duct in which the bluff body sits is three and six bluff body characteristic lengths, respectively, and extends $0.682 \mathrm{~m}$ downstream.

Laser Doppler Anemometry (LDA) was used to obtain velocity data. Both mean and RMS velocity data are available ${ }^{19}$ from measurements along the center-line and across the height of the combustor section, at several axial locations. Temperature and species concentration data are available ${ }^{20}$ from measurements using the Coherent AntiStokes Raman Scattering (CARS) technique.

\section{B. Codes}

Four codes are used for the simulation of the bluff body non-reacting and reacting flow fields, with solutions compared to each other and to experimental data. In order for a fair comparison to be made, the same physical subgrid models are used in all codes for closure of the turbulence and turbulence-chemistry interaction sub-grid terms. Since not all codes have the same modeling capabilities, the Smagorinsky ${ }^{37}$ and progress variable type combustion models are chosen due to the affordable levels of effort required for implementation, whilst providing an acceptable level of modeling fidelity. However, the numerical methods native to each code are used for the spatial discretization and temporal integration and hence the comparison of computational results can act as an investigation into the impact of numerical schemes, and hence numerical error, on the results obtained.

Two LES codes originating from leading academic institutions are employed in this study, namely CHARLES ${ }^{\mathrm{TM}}$ and LESLIE3D, along with the open source code OpenFOAM and commercial code Fluent.

\section{CHARLES}

The CHARLES code originates from Professor Parviz Moin's group at Stanford University but is currently developed and marketed by Cascade Technologies Inc. It is a density based unstructured finite-volume code which uses a central scheme for discretization of the Euler terms in the governing system of equations. This scheme is nominally second order accurate, but can be up to fourth order accurate on regular grids. For reacting solutions, this scheme is coupled to a second order essentially non-oscillatory (ENO) scheme for stabilization in high gradient regions of the flow, such as the flame. A second order accurate central scheme is used for discretization of the Laplacian terms in the governing system of equations. A third order TVD explicit Runge-Kutta scheme ${ }^{38}$ is used for temporal integration.

\section{$\underline{\text { LESLIE3D }}$}

The LESLIE3D code is developed by Professor Suresh Menon's group at Georgia Institute of Technology. It is a density based structured finite-volume LES code, and uses the $2^{\text {nd }}$ order predictor-corrector MacCormack scheme, which is central in nature for spatial discretization of the Euler fluxes and explicit in nature for temporal integration of the governing system of equations. However, the reacting solutions presented in this paper, unless otherwise stated, use the third order MUSCL scheme for the spatial discretization of these Euler fluxes. A second order accurate central scheme is used for discretization of the Laplacian terms in the governing system of equations.

\section{$\underline{\text { OpenFOAM }}$}

OpenFOAM is an open source code developed by OpenCFD Ltd. It is an unstructured finite-volume code which is capable of modeling a wide range of physical phenomena. A second order accurate bounded central scheme is used for the spatial discretization of the inviscid terms in the LES governing equations, which is bounded by a Sweby limiter. A second order accurate central scheme is used for discretization of the Laplacian terms in the governing system of equations. A second order accurate implicit (backward difference) scheme is used for the temporal integration and the PISO (pressure-implicit split-operator) is used for pressure-velocity coupling. The Green-Gauss node based method is chosen for gradient calculations. The same numerical schemes are used for both the non-reacting and reacting simulations and the pressure based solver is employed. 


\section{Fluent}

Fluent CFD is a commercially available code owned and marketed by Ansys Inc. Fluent is an unstructured finitevolume code which contains a wide range of numerical methods and models. A second order accurate bounded central scheme is used for spatial discretization of the Euler terms in the LES governing equations and a second order accurate central scheme is used for discretization of the Laplacian terms. Second order implicit time integration is employed and the PISO scheme is used for pressure-velocity coupling. The Green-Gauss node based method is chosen for gradient calculations and the PRESTO! scheme is employed for pressure interpolation. The same numerical schemes are used for both the non-reacting and reacting simulations and the pressure based solver is employed.

\section{Computational Models}

In compressible LES the governing system of instantaneous equations is Favre filtered, requiring the use of subgrid scale models for closure. The system of filtered equations is given by

$$
\begin{gathered}
\frac{\partial \bar{\rho}}{\partial t}+\frac{\partial \bar{\rho} \tilde{u}_{j}}{\partial x_{j}}=0 \\
\frac{\partial \bar{\rho} \tilde{u}_{i}}{\partial t}+\frac{\partial \bar{\rho} \tilde{u}_{i} \tilde{u}_{j}}{\partial x_{j}}=-\frac{\partial \bar{p}}{\partial x_{i}}+\frac{\partial \bar{\tau}_{i j}}{\partial x_{j}}-\frac{\partial \bar{\rho} \tilde{u}_{\imath}^{\prime} \tilde{u}_{j}^{\prime}}{\partial x_{j}} \\
\frac{\partial \bar{\rho} \tilde{E}}{\partial t}+\frac{\partial \bar{\rho} \tilde{u}_{j} \tilde{E}}{\partial x_{j}}=\frac{\partial}{\partial x_{j}}\left[\left(\frac{\tilde{\lambda}}{c_{p}}+\frac{\mu_{t}}{P r_{t}}\right) \frac{\partial \tilde{h}}{\partial x_{j}}\right]+\frac{\partial}{\partial x_{j}}\left[-\tilde{u}_{j} \bar{p}+\tilde{u}_{i} \bar{\tau}_{i j}\right] \\
\frac{\partial \bar{\rho} \tilde{C}}{\partial t}+\frac{\partial \bar{\rho} \tilde{u}_{j} \tilde{C}}{\partial x_{j}}=\frac{\partial}{\partial x_{j}}\left[\frac{\mu_{t}}{S c_{t}} \frac{\partial \tilde{C}}{\partial x_{j}}\right]+\overline{\dot{\omega}}_{C}
\end{gathered}
$$

where, $\bar{\rho}$ is the density, $\tilde{u}_{i}$ is the velocity and $\tilde{E}$ is the total energy. Since this study focuses on premixed combustion, the progress variable $(C)$ approach is employed, where $C=1$ corresponds to pure products and $C=0$ corresponds to pure reactants.

The Smagorinsky model is employed for closure of the $\bar{\rho} \widetilde{u_{\imath}^{\prime}} u_{\jmath}^{\prime}$ term, through use of the Boussinesq approximation:

$$
-\bar{\rho} \widetilde{u_{\imath}^{\prime} u_{\jmath}^{\prime}}=2 \mu_{t}\left(\tilde{S}_{i j}-\frac{1}{3} \frac{\partial \tilde{u}_{k}}{\partial x_{k}} \delta_{i j}\right)-\frac{2}{3} \bar{\rho} k \delta_{i j}
$$

where the sub-grid dynamic viscosity is

$$
\mu_{t}=\bar{\rho}\left(C_{s} \Delta\right)^{2}\left|\sqrt{2 \tilde{S}_{i j} \tilde{S}_{i j}}\right|
$$

with the strain rate tensor defined as

$$
\tilde{S}_{i j}=\frac{1}{2}\left(\frac{\partial \tilde{u}_{j}}{\partial x_{i}}+\frac{\partial \tilde{u}_{i}}{\partial x_{j}}\right)
$$


The model constant, $C_{s}$, is set to 0.2 in all simulations presented in this paper. $\Delta$ is the filter width, defined as (cell volume $)^{1 / 3}$.

In order to close the reaction rate term in the Favre filtered progress variable transport equation a sub-grid scale combustion model is required. Here, the flame surface density (FSD) model of Weller et al. ${ }^{39}$ is employed:

$$
\overline{\dot{\omega}}_{C}=\rho_{r} S_{L} \Sigma_{g e n}
$$

where, $\rho_{r}$ is the density of the reactants and $S_{L}$ is the laminar flame speed. $\Sigma_{g e n}$ is the generalized filtered flame surface density, defined as

$$
\Sigma_{g e n}=\overline{|\nabla C|}
$$

which requires modeling:

$$
\Sigma_{g e n}=[1+2 \tilde{C}(\theta-1)]|\nabla \tilde{C}|
$$

with

$$
\theta=1+0.62 \sqrt{\frac{u_{\Delta}^{\prime}}{S_{L}}} \operatorname{Re}_{\eta}
$$

where

$$
\operatorname{Re}_{\eta}=\frac{u_{\Delta}^{\prime} \eta}{v}=\frac{u_{\Delta}^{\prime}}{(v \varepsilon)^{1 / 4}}
$$

for $\varepsilon=C_{\varepsilon} \frac{u_{\Delta}^{\prime 3}}{\Delta}$ and $C_{\varepsilon}=1.048$. The complexity of the model is in the definition of $u_{\Delta}^{\prime}$, which is based on the strain rate tensor. $u_{\Delta}^{\prime}$ is given by

$$
u_{\Delta}^{\prime}=\sqrt{2 k_{s g s}}
$$

where

with

$$
k_{s g s}=u_{s g s}^{2}=\left(\frac{-b+\sqrt{b^{2}+4 a c}}{2 a}\right)^{2}
$$

$$
a=\frac{C_{\varepsilon}}{\Delta} \quad b=\frac{2}{3}\left[\operatorname{trace}\left(\tilde{S}_{i j}\right)\right] \quad c=2 C_{k} \Delta\left[\operatorname{dev}\left(\tilde{S}_{i j}\right): \tilde{S}_{i j}\right]
$$

where $\operatorname{dev}\left(\tilde{S}_{i j}\right)$ is the deviatoric part of the strain rate tensor, defined as

$$
\tilde{S}_{i j}-\frac{1}{3}\left[\operatorname{trace}\left(\tilde{S}_{i j}\right)\right] I
$$

The model constant $C_{k}$ is set to 0.02 .

Two different methods are used for the thermodynamic coupling. CHARLES, OpenFOAM and Fluent assume adiabatic conditions, bypassing the energy equation, where temperature is defined by:

$$
T=T_{r}(1-C)+T_{p} C
$$


Here, $T_{r}$ and $T_{p}$ are the temperature of the reactants and products, respectively, defined by boundary conditions and composition. $T_{r}$ and $T_{p}$ are respectively taken as $288 \mathrm{~K}$ and $1784 \mathrm{~K}$, here. LESLIE3D assumes a calorically perfect gas, and obtains the temperature from the energy equation:

$$
T=\frac{\left(E-\frac{1}{2} u_{i}^{2}-h_{\text {mix }}^{o}\right)}{c_{v, \text { mix }}}
$$

where, $h_{\text {mix }}^{o}$ and $c_{v \text {,mix }}$ are the mixture values for enthalpy of formation and specific heat at constant volume, respectively. Since the mixture gas constant can be defined as:

$$
R_{\text {mix }}=R_{r}(1-C)+R_{p} C
$$

where, $R_{r}$ and $R_{p}$ are the reactant and product gas constants, respectively, pressure can be obtained from the ideal gas law since density is known from the continuity equation.

The intent of this work was to maintain identical physical models between all codes used. The above difference in thermodynamic coupling between LESLIE3D and the other codes is the only violation of this paradigm and was necessitated due to implementation constraints. It is considered acceptable under the assumption that the operating conditions and relatively simple mixture compositions considered in this work are such, that the difference between the two approaches should be very small. This assumption is verified in Figure 1 which compares the adiabatic and calorically perfect methods used for thermodynamic coupling by plotting the temperature as a function of progress variable. The linear function of the adiabatic approach is compared to a linear fit through simulation results of the Volvo case using the LESLIE3D code. The very close agreement between the two approaches shown in Figure 1 provides confidence that the uncertainty associated with comparing computational results from LESLIE3D with the other codes is very small.

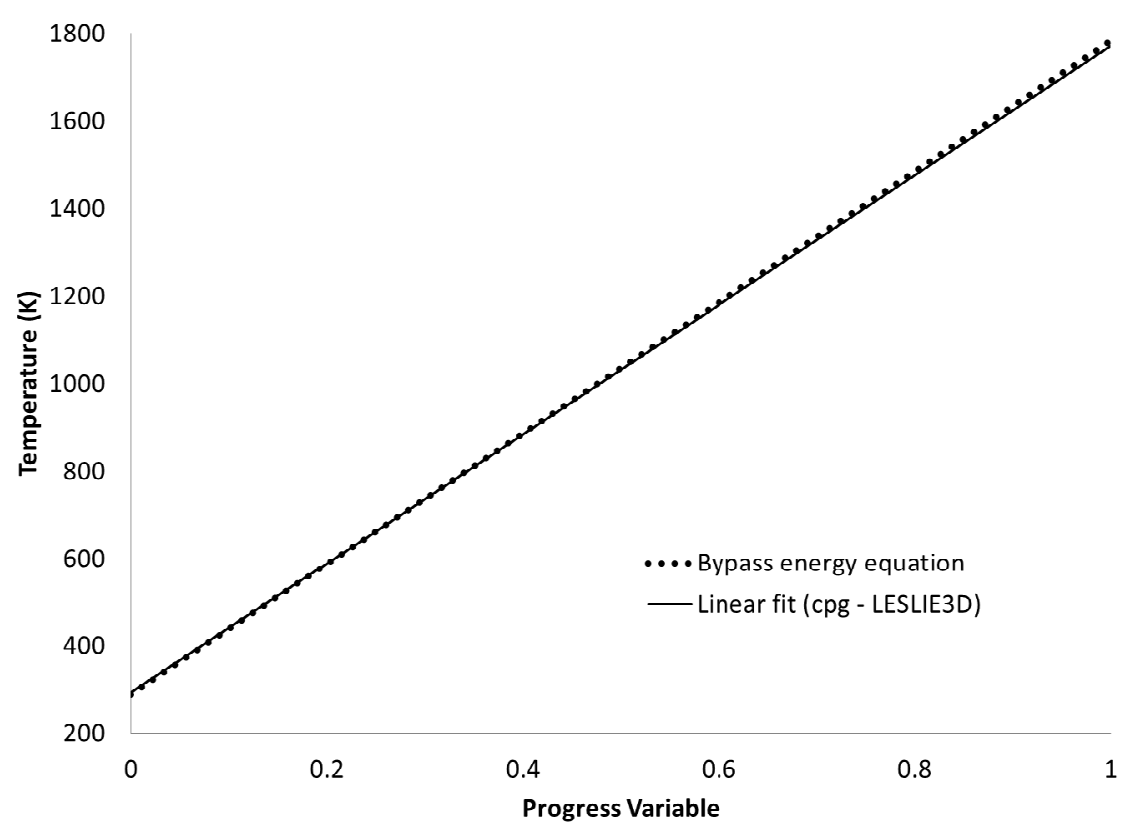

Figure 1: Comparison of methods used for thermodynamic coupling

\section{Computational Grid}

The grids employed in this study were chosen carefully by estimating several physical length scales that play a role in turbulent mixing and combustion. The boundary layer on the bluff-body plays an important role in the formation of the shear layer, and its thickness ${ }^{40}$ is proportional to $\left(1 / \sqrt{ } \operatorname{Re}_{\mathrm{D}}\right)$. Here $\operatorname{Re}_{\mathrm{D}}$ is the bulk Reynolds number based on the bluff-body height $(D)$ and the inflow velocity $\left(U_{o}\right)$. This gives an estimate of $5 \mathrm{~mm}$. A Kolmogorov 
length scale can also be estimated from this Reynolds number and length scale, at $12 \mu \mathrm{m}$. However, the Kolmogorov length scale does not need to be resolved on a LES mesh and hence should not control the required grid resolution.

Another estimate for the required grid resolution can be based on the filter size requirement in LES. Even though there is no theoretical justification, it is generally accepted that in LES, at least $80 \%$ of the turbulent kinetic energy ${ }^{1}$ must be resolved so that the bulk of the energy containing motions are directly captured. Based on this criterion, Pope $^{1}$ shows that a filter-width to integral length scale ratio of $(\Delta / \mathrm{L}) \approx 0.083$ would resolve at least $80 \%$ of the kinetic energy in non-reacting high-Reynolds number homogeneous isotropic turbulence, when using a sharp spectral filter. Here, $\mathrm{L}$ is a measure of the flow length scale that is related to the integral scales of motion and by using $\mathrm{L}=\mathrm{D}$ (bluff-body characteristic dimension), we can estimate the filter width as $\Delta \approx 3 \mathrm{~mm}$. Even though this resolution may resolve $80 \%$ of the energy away from the walls, it may not resolve the near wall structures in the viscous sub-layer. Therefore, grid clustering near the walls must be used to resolve at least the log-layer region of the turbulent boundary layer.

Since Pope's criterion was developed for non-reacting high Reynolds number homogenous isotropic turbulence, it may not be appropriate for the approximation of the resolution required to capture $80 \%$ of the kinetic energy in a reacting flow. Caution should also be taken in application of Pope's criterion to define the grid resolution required by different codes, since the impact of numerical error is not taken into account. One code employing a more dissipative scheme may require a finer grid than a code employing a less dissipative scheme, to achieve the same level of accuracy. Pope's criterion also makes assumptions about the use of a sharp spectral filter function and the presence of a Kolmogorov spectrum. Such filter shapes are generally not used in traditional LES since the solution is implicitly filtered by the grid, and the possible presence of combustion generated turbulence means the assumption of a Kolmogorov spectrum is unlikely to hold. Knowledge and understanding of such physical processes is required in order to quantify the levels of sub-grid kinetic energy in a reacting flow and hence estimate the grid resolution required to capture $80 \%$ of the total kinetic energy. However, it is possible that even the requirement to capture at least $80 \%$ of the kinetic energy may need to be revised for a reacting flow. In general, additional criteria are required to help define the mesh resolution needed for such simulations. Due to a lack of such criteria, the grid resolution validated for the non-reacting case will be used for the reacting simulations.

Based on the above arguments, three non-uniform grids are generated, in order for a grid convergence study to be conducted. Points are clustered near the walls, with the minimum wall normal distance equal to $0.3 \mathrm{~mm}$, and stretched gradually to the 'free-stream' resolution. Due to Pope's criterion giving a required cell size of $3 \mathrm{~mm}$, three grid resolutions of $4 \mathrm{~mm}, 2 \mathrm{~mm}$ and $1 \mathrm{~mm}$ are generated in order to investigate the validity of this estimation. The grids employed are nearly isotropic in most regions (to minimize the commutative errors produced by non-uniform grids), except near the walls, as shown in Figure 2. The majority of results presented in this paper are for the $2 \mathrm{~mm}$ resolution grid, with the $4 \mathrm{~mm}$ and $1 \mathrm{~mm}$ results used to support the discussion, when required.

All solutions presented are on grids with a depth of two bluff-body widths, corresponding to one third of the tunnel dimension, with periodic boundary conditions employed in this direction. The grid accounts for the full length of the combustor downstream of the bluff body, extending $0.1 \mathrm{~m}$ upstream and $0.682 \mathrm{~m}$ downstream of the bluff body. No-slip boundary conditions are applied to the bluff body and top and bottom walls of the combustor duct. The total cell count for the $4 \mathrm{~mm}, 2 \mathrm{~mm}$ and $1 \mathrm{~mm}$ grids is 0.31 million, 1.76 million and 10.5 million cells, respectively.

Comparing the LES mesh resolution requirements to the Kolmogorov length scale gives an indication of the cost benefits of LES over DNS. Taking the $2 \mathrm{~mm}$ grid resolution, a cell size at least 167 times smaller would be required to capture the Kolmogorov length scales, corresponding to approximately $8.2 \times 10^{12}$ cells in $3 \mathrm{D}$, which is beyond current computational capabilities.

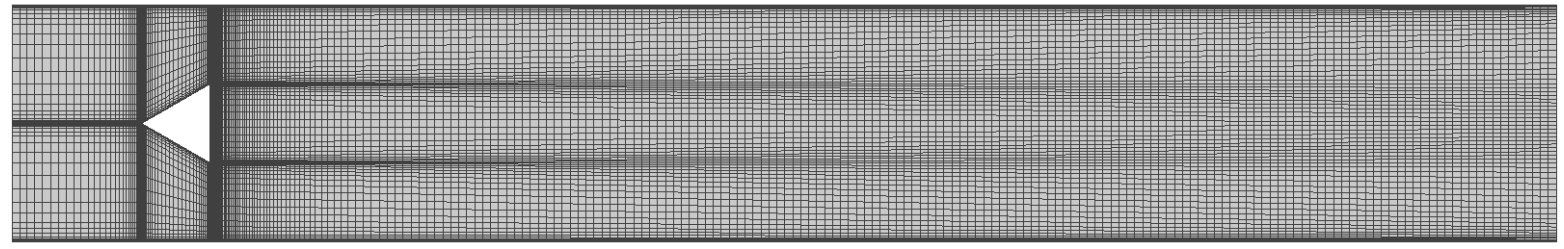

Figure 2: Computational grid (4mm mesh) showing clustering near walls and isotropic mesh away from walls

Temporal resolution is as important as spatial resolution in order to avoid excessive numerical dissipation and numerical instability. Physical time scale estimates are used to obtain the minimum time resolution requirements for a simulation. Vortex shedding is a dominant feature in non-reacting bluff-body flows and the experiment reports a 
shedding frequency of $105 \mathrm{~Hz}^{19}$. Based on this, a shedding time scale of $\Delta \mathrm{t}_{\text {shedding }}=9.5$ milliseconds, is obtained. The integral time scale $\left(\mathrm{t}_{\mathrm{I}}\right)$ and Kolmogorov time scale $\left(\mathrm{t}_{\eta}\right)$ are estimated using the corresponding length scales and the experimentally measured turbulence intensity in the shear layer. This yields $t_{I}=4$ milliseconds and $t_{\eta}=0.02$ milliseconds. However, since Kolmogorov length scales are not captured on a LES grid, this estimation of the corresponding time scale need not act as a strict limitation on the time step. Based on the speed of sound at a temperature of $288 \mathrm{~K}$, the inlet velocity and $2 \mathrm{~mm}$ cell size, a CFL criterion limited time step can be estimated at 0.006 milliseconds. However, deviations from this CFL based calculation may exist in some regions of the grid, due to locally finer resolution, acceleration above the inlet velocity and a higher speed of sound in the reacting case due to the presence of hot products.

Based on these estimates, a conservative value of 0.001 milliseconds or below is chosen as the target timeresolution for the simulations. Due to CFL restrictions associated with some of the numerical schemes employed, along with acceleration and high temperatures associated with the reacting flow, the actual time step sizes for each code are presented in Table 1. Although the use of different time steps in different codes could introduce numerical error into the solution, this is assumed to be small since all time steps remain well below any physical time scales captured in the simulations.

\begin{tabular}{lll}
\hline Code & Non-reacting & Reacting \\
\hline CHARLES & $1.0 \times 10^{-6} \mathrm{~s}$ & $3.0 \times 10^{-7} \mathrm{~s}$ \\
LESLIE3D & $6.0 \times 10^{-7} \mathrm{~s}$ & $1.4 \times 10^{-7} \mathrm{~s}$ \\
OpenFOAM & $1.0 \times 10^{-6} \mathrm{~s}$ & $3.0 \times 10^{-7} \mathrm{~s}$ \\
Fluent & $1.0 \times 10^{-6} \mathrm{~s}$ & $1.0 \times 10^{-6} \mathrm{~s}$ \\
\hline
\end{tabular}

Table 1: Time steps used with each code

The majority of simulations presented in this paper were run on computational resources internal to United Technologies Research Center. However, a $1 \mathrm{~mm}$ resolution reacting simulation for CHARLES was run on 30,000 cores of the Cray XK6 machine at the Oak Ridge Leadership Computing Facility.

\section{Results and Discussion}

\section{A. Non-reacting Simulations}

Results from non-reacting simulations are presented on the $4 \mathrm{~mm}$ and $2 \mathrm{~mm}$ resolution grids, in order to establish the validity of the LES models and grid topology employed. The non-reacting flow field around a bluff body is characterized by large coherent vortices, shedding in an asymmetric manner at a characteristic frequency. Figure 3 shows iso-surfaces of vorticity magnitude colored by the z-component of vorticity for a simulation from CHARLES, on the $2 \mathrm{~mm}$ resolution grid. Coherent vortices can be clearly seen convecting downstream, produced by the VonKarman like shedding behind the bluff body. It can also be seen that additional vortices are shed from the upper and lower walls of the combustor duct, due to regions of separation caused by the presence of an adverse pressure gradient. In fact, it is these wall vortices and their interaction with the primary vortices shed from the bluff body which leads to a rapid three dimensional breakdown, generating smaller turbulent structures further downstream.



Figure 3: Isosurfaces of vorticity magnitude at levels $2000 \mathrm{~s}^{-1}$ and $4000 \mathrm{~s}^{-1}$ for a non-reacting simulation from CHARLES on the $2 \mathrm{~mm}$ resolution grid, colored by the $\mathrm{Z}$ component of vorticity. 
It can be seen from Figure 4 that the results on the $4 \mathrm{~mm}$ grid are in reasonable agreement with experimental data for the mean centerline axial velocity, anisotropy and fluctuation levels, for most codes. The anisotropy and fluctuation level are respectively defined as:

$$
\frac{v^{\prime}}{u^{\prime}} \quad \frac{\sqrt{u^{\prime 2}+v^{\prime 2}}}{U_{b u l k}}
$$

with $U_{\text {bulk }}$ corresponding to the inlet velocity of $17 \mathrm{~m} / \mathrm{s}$.

LESLIE3D predicts acceleration along the centerline on the $4 \mathrm{~mm}$ resolution grid, which can clearly be seen from the mean axial velocity contours in Figure 5, where the results from CHARLES and LESLIE3D are compared. From the instantaneous images in Figure 6, it can be seen that the solution from LESLIE3D contains more coherent vortices. It should be noted that similar solutions were seen with all the codes in two-dimensional simulations of the same problem ${ }^{41}$. It was postulated that this type of solution manifests itself when three-dimensional vortex breakdown of the wake vortices is impaired. Figure 6 clearly indicates that this is the case for LESLIE3D even in the 3D environment. This may suggest slightly higher levels of numerical dissipation or lower levels of numerical noise to aid vortex breakdown in this code. Returning to Figure 4, it can be seen that this centerline acceleration leads to larger values for the anisotropy and fluctuation level for LESLIE3D, than for the other codes. 

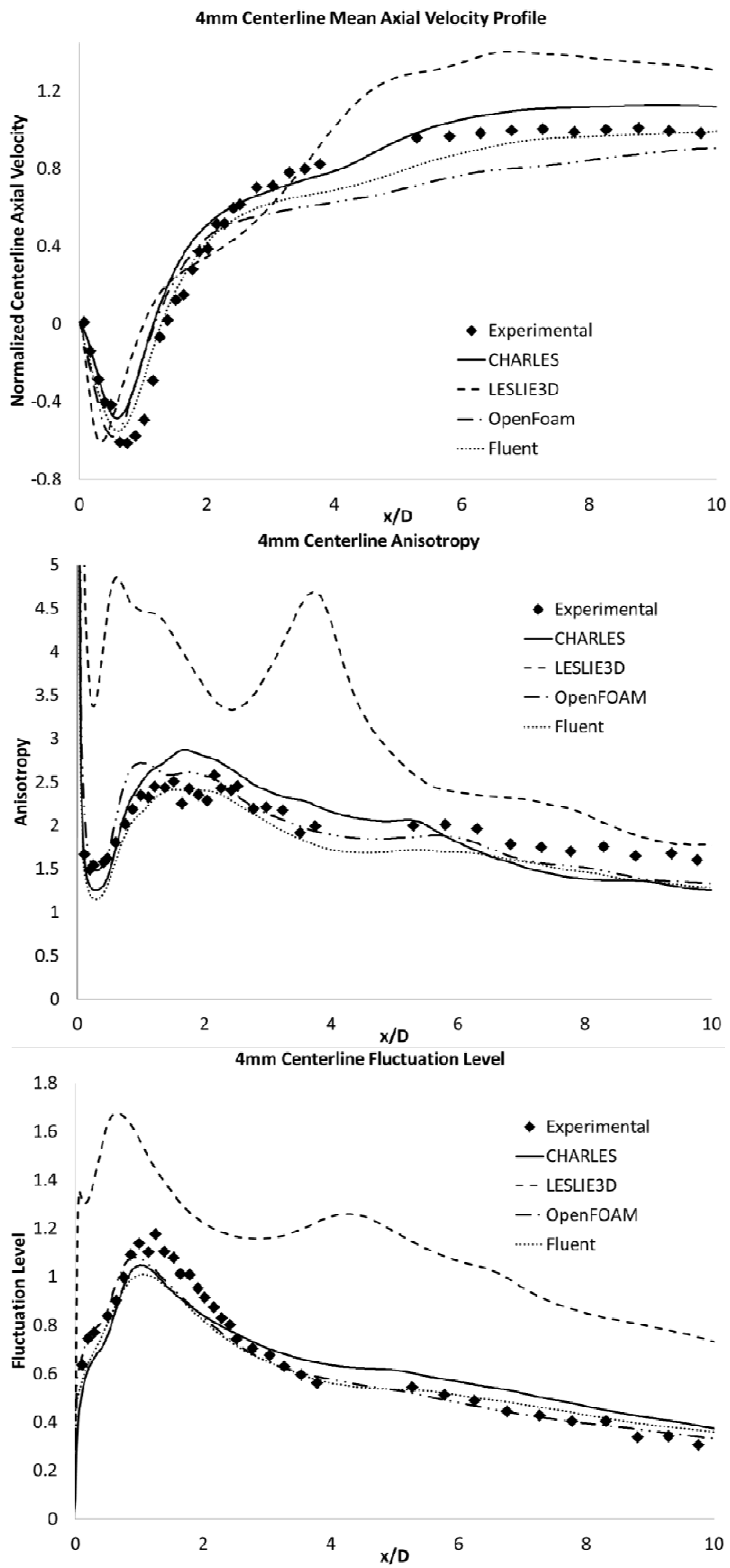

Figure 4: Centerline profiles for the mean axial velocity, anisotropy and fluctuation level on the $4 \mathrm{~mm}$ resolution grid for the non-reacting case. 

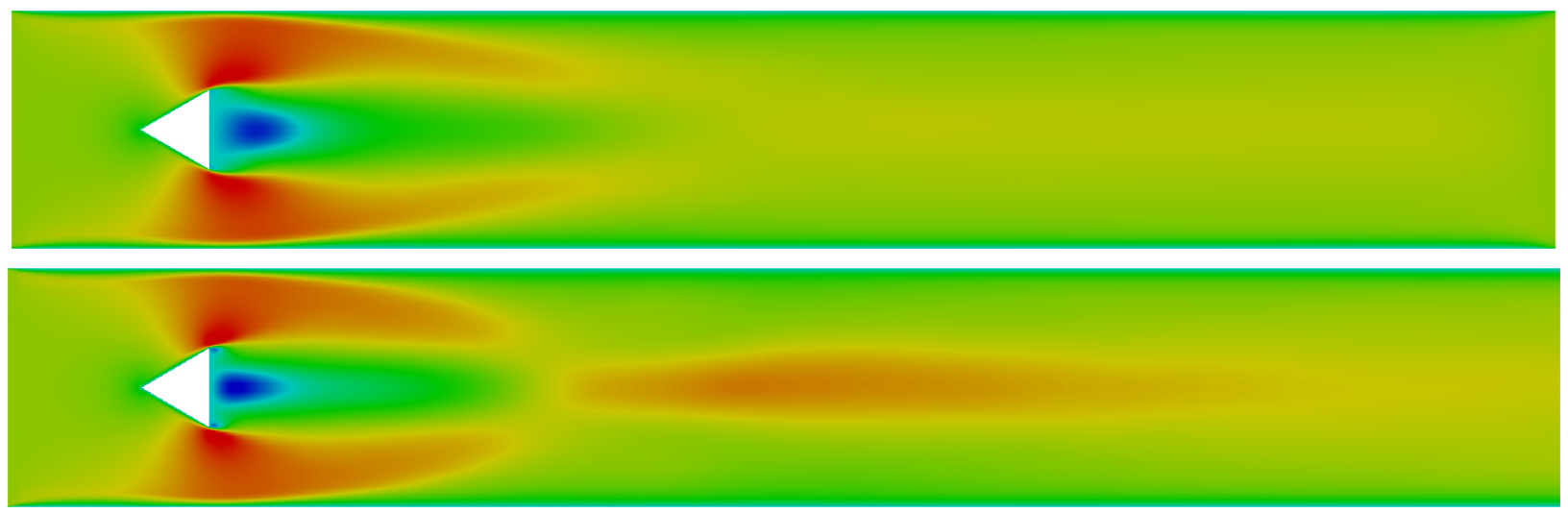

Figure 5: Comparison of mean axial velocity contours from CHARLES (top) and LESLIE3D (bottom) on the $4 \mathrm{~mm}$ resolution grid, for the non-reacting case.

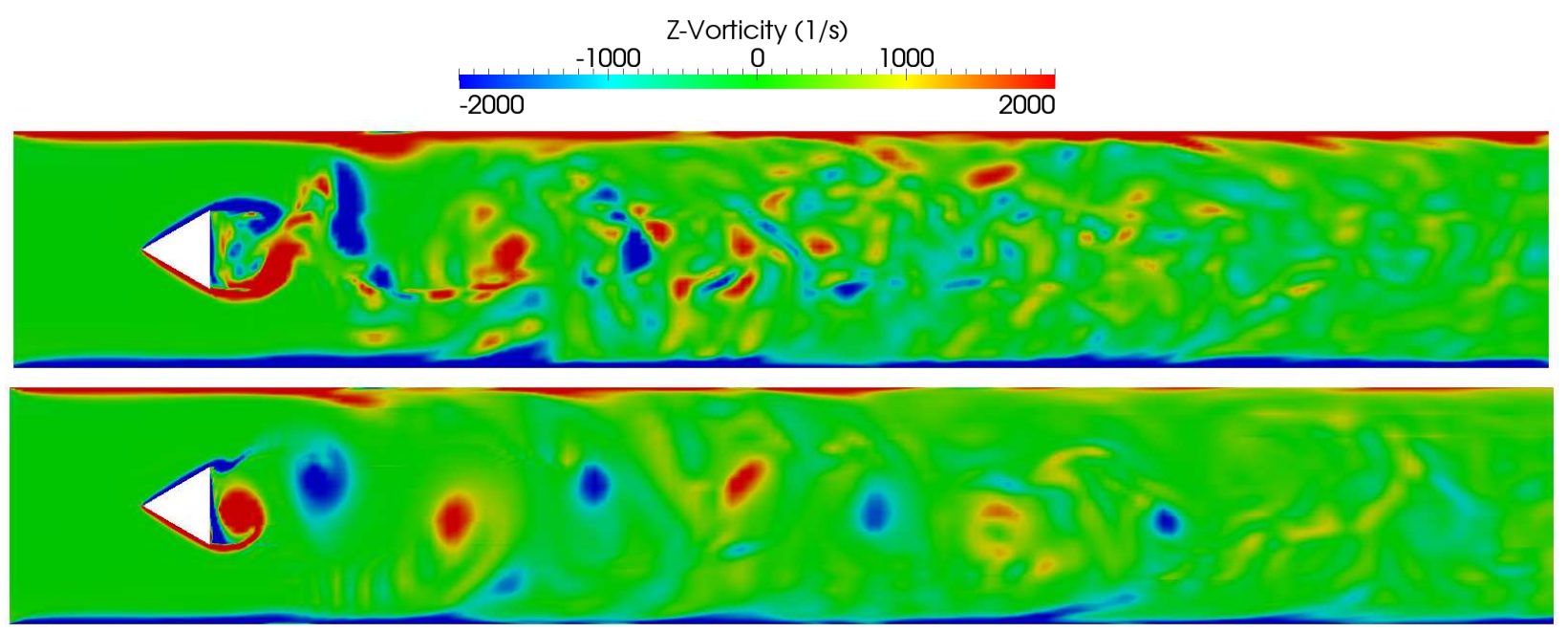

Figure 6: Comparison of instantaneous z-vorticity contours from CHARLES (top) and LESLIE3D (bottom) on the $4 \mathrm{~mm}$ resolution grid, for the non-reacting case.

However, the analysis conducted to determine cell size requirements resulted in a $3 \mathrm{~mm}$ resolution grid being needed in order to directly capture $80 \%$ of the turbulent kinetic energy. The results from all codes, from simulations on a grid that obeys this constraint $(2 \mathrm{~mm})$, are presented in Figure 7 . It can be seen that all codes converge to the same results, giving good agreement with experimental profiles. The transition between solutions experienced by the LESLIE3D code with change of grid from $4 \mathrm{~mm}$ to $2 \mathrm{~mm}$ demonstrates the potentially significant impact of numerical error on the solution near flow transition regions. It also motivates the need for the definition of physicsbased criteria for numerical resolution determination. 



Figure 7: Centerline profiles for the mean axial velocity, anisotropy and fluctuation level on the $2 \mathrm{~mm}$ resolution grid for the non-reacting case. 
Figure 8 and Figure 9 respectively show the normalized mean axial velocity and normalized mean Reynolds stress profiles across the combustor duct at five axial locations, for all codes, on the $2 \mathrm{~mm}$ resolution grid. The mean Reynolds stress is defined as:

$$
\frac{\overline{u^{\prime} v^{\prime}}}{U_{b u l k}^{2}}
$$

Again, it can be concluded that the results from each non-reacting simulation are in reasonable agreement with the experimental data, confirming the $2 \mathrm{~mm}$ resolution to be sufficient for the non-reacting flow field. This demonstrates a validation of each code for this test case and demonstrates that the physical sub-grid model employed is sufficient to accurately capture the physics associated with this non-reacting flow.
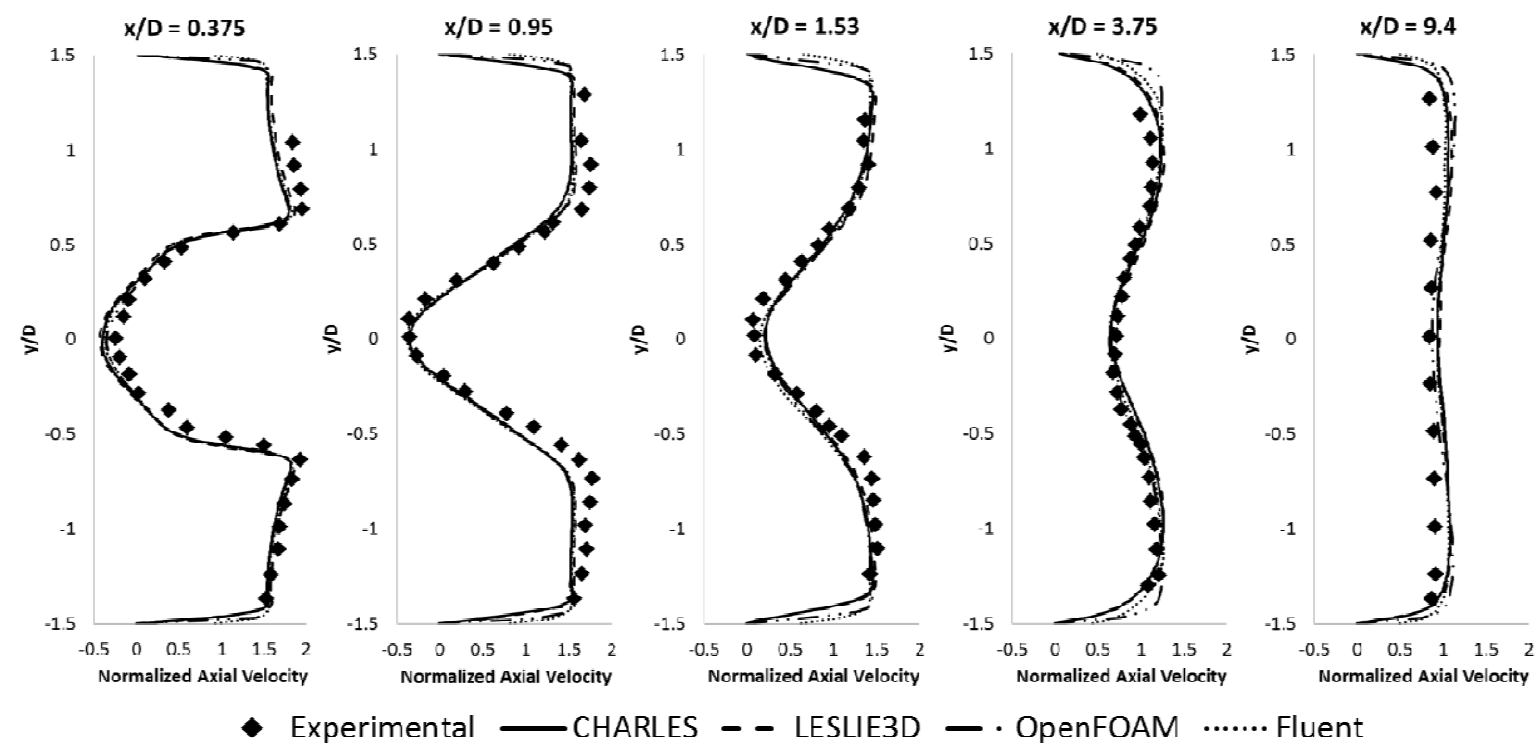

Figure 8: Normalized mean axial velocity wake profiles on the $2 \mathrm{~mm}$ grid, at several axial (x) locations for the non-reacting case.
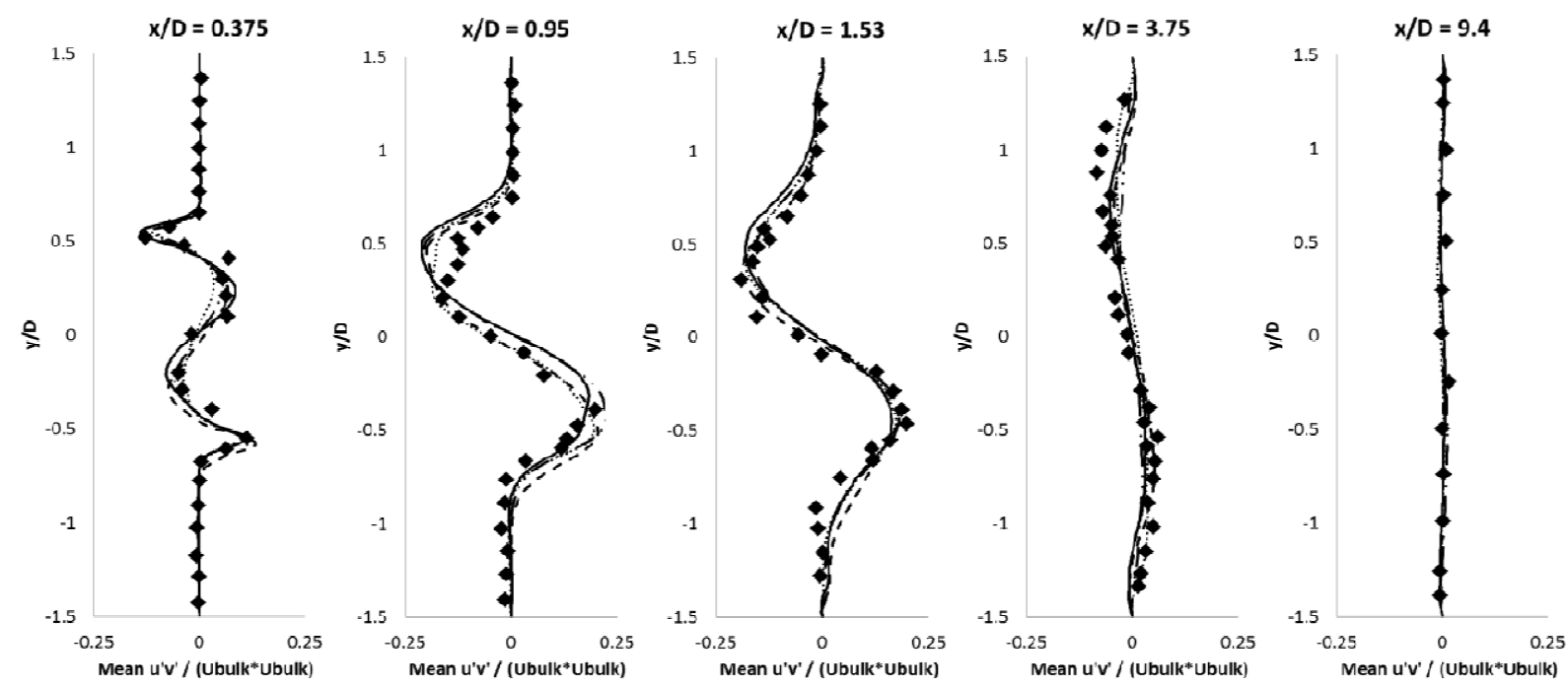

Experimental

CHARLES

S - - LESLIE3D - OpenFOAM ….... Fluent

Figure 9: Normalized mean Reynolds stress wake profiles on the $\mathbf{2} \mathbf{m m}$ grid, at several axial $(x)$ locations for the non-reacting case. 
The computational results can be used to check the method used to calculate the grid resolution; specifically, the requirement to resolve at least $80 \%$ of the total kinetic energy. The percentage of resolved kinetic energy can be calculated from

$$
\frac{\bar{u}_{i} \bar{u}_{i}}{\bar{u}_{i} \bar{u}_{i}+u_{\Delta}^{\prime} u_{\Delta}^{\prime}}
$$

where, the numerator corresponds to the total resolved kinetic energy and the denominator corresponds to the total kinetic energy. However, the sub-grid kinetic energy $\left(u_{\Delta}^{\prime} u_{\Delta}^{\prime}\right)$ is not directly available and hence the model in Equation (13) is used to calculate the sub-grid fluctuating velocity, here. Instantaneous contours of this percentage of resolved kinetic energy are provided in Figure 10, for both the $4 \mathrm{~mm}$ and $2 \mathrm{~mm}$ resolution results, from each code. It is suggested from these contours that both grids, for all codes, capture much more than $80 \%$ of the total kinetic energy in the vast majority of the domain. Since the cell sizes do not correspond to DNS resolution this is highly unlikely, particularly on the $4 \mathrm{~mm}$ resolution grid. Therefore, the model employed here for the sub-grid fluctuating velocity may under predict the sub-grid kinetic energy. This suggests our estimate of the sub-grid kinetic energy from resolved quantities using the Weller model is not reliable. A better estimation of the sub-grid kinetic energy in the non-reacting flow may be obtained by using a more advanced sub-grid-scale model, such as a $\mathrm{k}_{\mathrm{sgs}}$ sub-grid-scale model. However, it is likely neither approach will provide a correct measure of the sub-grid kinetic energy when combustion is present.

Despite these issues, it can be concluded from Figure 10 that the $2 \mathrm{~mm}$ resolution grid does capture a higher percentage of the total kinetic energy, particularly for LESLIE3D (as would also be suggested by the results above), and the near wall regions are correctly identified as under-resolved parts of the grid.



Figure 10: Instantaneous contours of percent of resolved kinetic energy, on both the $4 \mathrm{~mm}$ and $2 \mathrm{~mm}$ resolution grids, for the non-reacting simulations from (top to bottom): CHARLES, LESLIE3D, OpenFOAM and Fluent.

\section{A. Reacting Simulations}

It has been demonstrated that all four codes can accurately predict the non-reacting flow field for the Volvo test case on a $2 \mathrm{~mm}$ grid, even though a simple turbulence sub-grid scale model is used and the numerical schemes in each code differ. Results from reacting simulations from each code are presented in this section in order to investigate whether accurate predictions are still obtained when the complexity of combustion is added into the flow.

In the absence of additional constraining resolution criteria for reacting flow simulation, the $2 \mathrm{~mm}$ resolution grid that was demonstrated to be sufficient for the non-reacting flow field is employed for the reacting simulations. Therefore, all results presented will be for this grid, unless otherwise stated. As previously discussed, the reacting 
simulations are performed by solving a progress variable transport equation with a flame-surface density based combustion model.

It has been shown both numerically $y^{29}$ and experimentally ${ }^{36}$ for a premixed bluff-body stabilized flame that if the density ratio between reactants and products is high enough, the heat release leads to a suppression of the asymmetric vortex shedding which is characteristic of the non-reacting flow field. This leads to a symmetric flame shape at high heat release; a significant switch in flow physics from the non-reacting to reacting flow fields, which the turbulence and combustion sub-grid models must be capable of capturing. This transition from asymmetric to symmetric behavior was shown to occur between density ratios of 1.7 and 2.4. The density ratio simulated here for the Volvo test case is approximately 6, suggesting that the flow is far from the solution transition region and should experience a robustly symmetric flame. This is supported by experimental observations, where it was reported that no characteristic frequency of oscillation consistent with asymmetric bluff-body shedding existed ${ }^{19}$.

Although a symmetric flame is the correct physical solution to this problem, accurately capturing this behavior presents a significant challenge to the sub-grid models. As stated in the introduction, many simulation results for this reacting test case present in the literature ${ }^{24,27,28,31,32,33}$ show an asymmetric flame.

Figure 11 shows typical iso-surfaces of vorticity magnitude, colored by the z-component of vorticity, from CHARLES. It can clearly be seen that the reacting flow field is strikingly different from the non-reacting flow field shown in Figure 3. Vortex shedding is indeed suppressed and a symmetric flame exists downstream of the bluff body, at least at the time instant shown here. Another interesting feature which can be observed from Figure 11 is that no vortices can been seen shedding from the upper and lower walls of the combustor and the interaction of the wake with the walls is significantly lower than in the non-reacting case. This suggests that the interaction of the wall boundary layers with the wake in the reacting simulations is not as important.

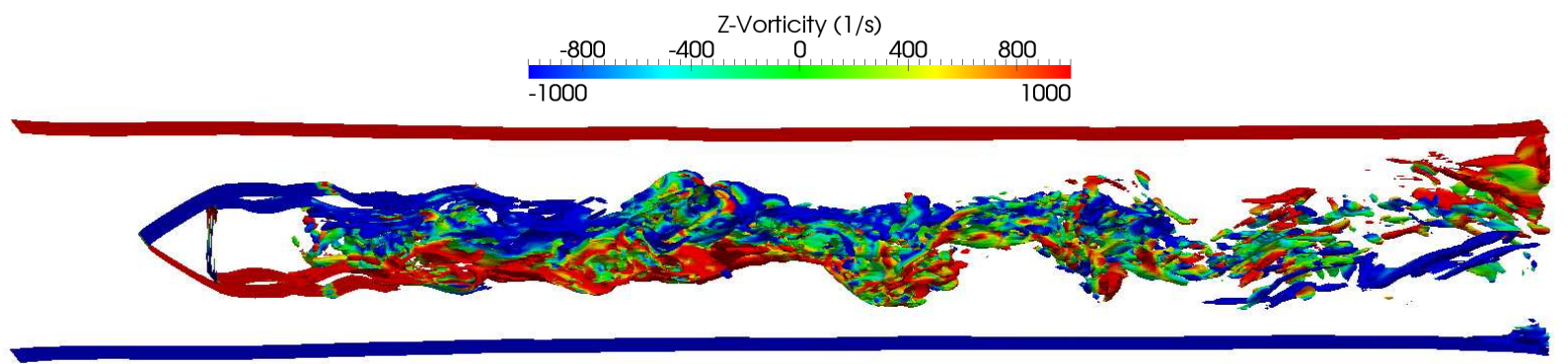

Figure 11: Isosurfaces of vorticity magnitude at level $2000 \mathrm{~s}^{-1}$ for the reacting simulation from CHARLES, colored by the $\mathrm{Z}$ component of vorticity.

Figure 12 shows typical instantaneous temperature contours from the four codes in question. It can be seen that, at the time instants shown, CHARLES, LESLIE3D and Fluent all predict a symmetric flame shape for this test case, whilst OpenFOAM produces an asymmetric solution. For such qualitative analysis, experimental flow field visualizations would be extremely valuable, but are not available in the literature for this test case. With the wider application of time accurate simulation techniques today, such as LES, it should become standard practice to obtain high quality flow field visualizations in modern experiments. The qualitative agreement of a computational solution with the experiment can then be verified.

It turns out that the OpenFOAM solution is permanently asymmetric and it can clearly be seen from Figure 13 that the sinusoidal nature of the OpenFOAM flame leads to a large acceleration downstream, which is not in agreement with experimental data. The possibility of physical sub-grid model implementation error by the authors was not considered since OpenFOAM is the only code used in this study where no modifications to the source code were required. This is because the Smagorinsky and Weller sub-grid models are included in the standard public release version of the code. The asymmetric shape of the flame implies that a larger volume of the duct contains hot products than should be the case, leading to a larger heat release and hence over acceleration of the flow. The source of this poor agreement with experimental data for the mean centerline axial velocity also leads to poor results for second order statistics and yields a dominant frequency due to the sinusoidal motion of the flame (not seen in the experiment). Therefore, no further results will be presented from the OpenFOAM simulations.

It should be noted, however, that OpenFOAM results for this reacting Volvo test case are available in the literature $^{27}$ which show better agreement with the experimental data in terms of mean velocity and temperature profiles and related statistics. A different combustion model, turbulence sub-grid scale model and numerical scheme was used in the referenced study. An asymmetric flame shape was still observed, however, which is inconsistent with the experimental evidence. This kind of behavior, i.e. the ability to be in agreement with experimental 
quantitative results, while missing the actual dynamic behavior of the flow has also been seen in other studies employing other codes and models, as indicated in the Introduction. It suggests that requirements for what represents 'reasonable agreement' have to become more conservative. It also implies that more detailed experimental measurements are likely required for comprehensive validation, including flow field visualization.
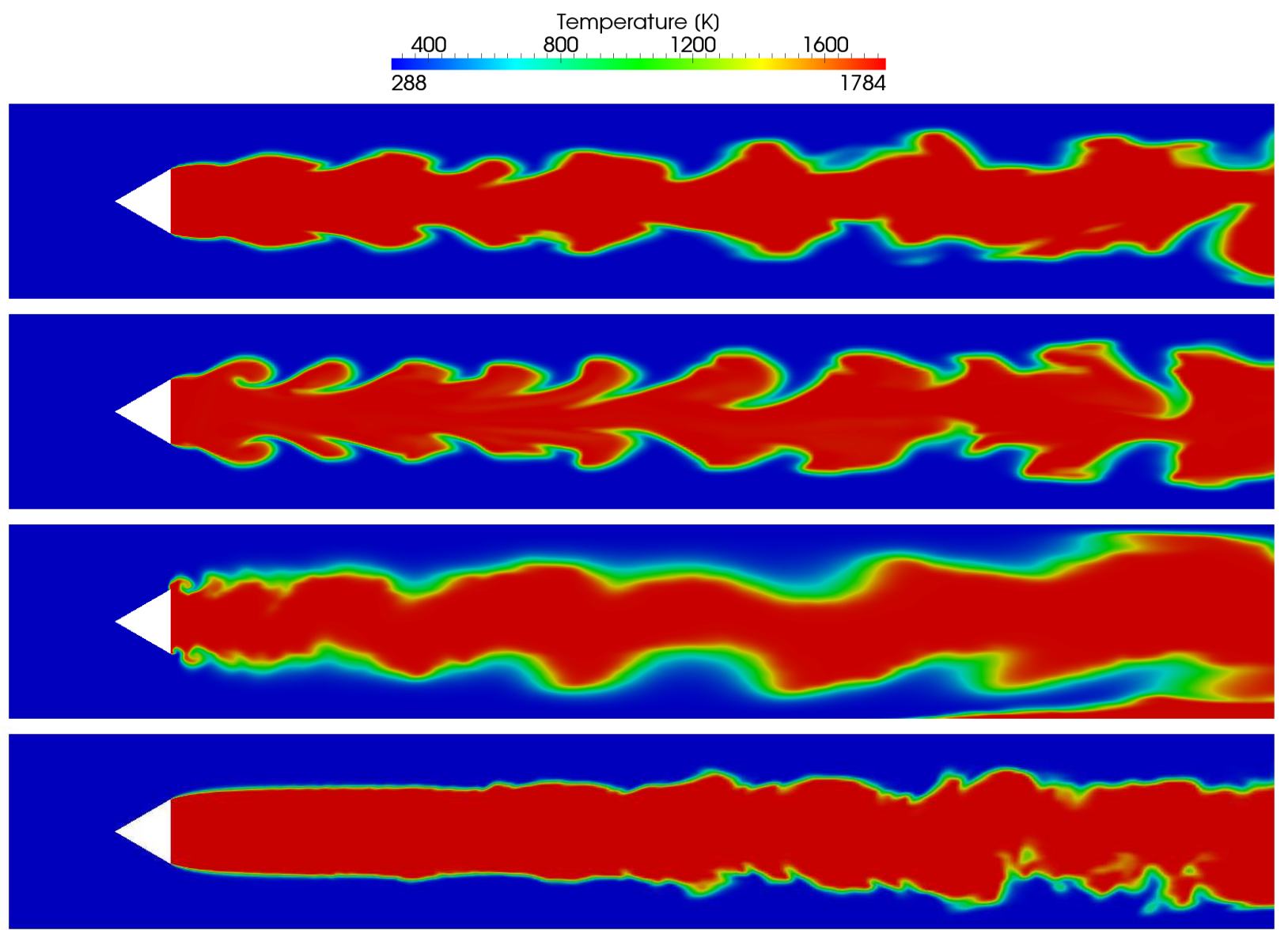

Figure 12: Instantaneous temperature contours. Top to bottom: CHARLES, LESLIE3D, OpenFOAM and Fluent.

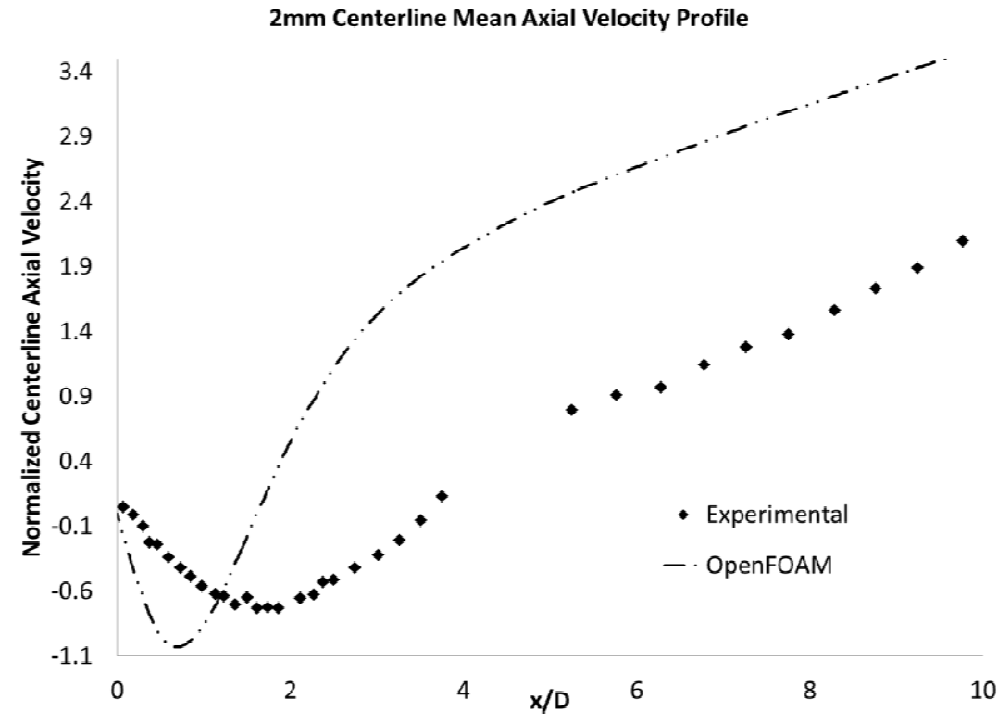

Figure 13: Mean centerline axial velocity profile on the $2 \mathrm{~mm}$ grid for the reacting solution from OpenFOAM. 
Although the images in Figure 12 show a symmetric flame shape for CHARLES, LESLIE3D and Fluent, the solutions do occasionally exhibit a sinusoidal motion. Snapshots of this motion are presented in Figure 14. As already noted, the physical transition from asymmetric to symmetric behavior is supposed to occur at much lower density ratios, namely between 1.7 to 2.4 . The modeling ${ }^{29}$ and experimental ${ }^{36}$ studies that defined this range also pointed out that occasional asymmetric events can also occur outside this range but are increasingly rare. Specifically, it was found ${ }^{36}$ that the 'sinusoidal event' arrival rate and event duration both decrease with increasing density ratio, becoming rare by 3.4 (the maximum density ratio tested).

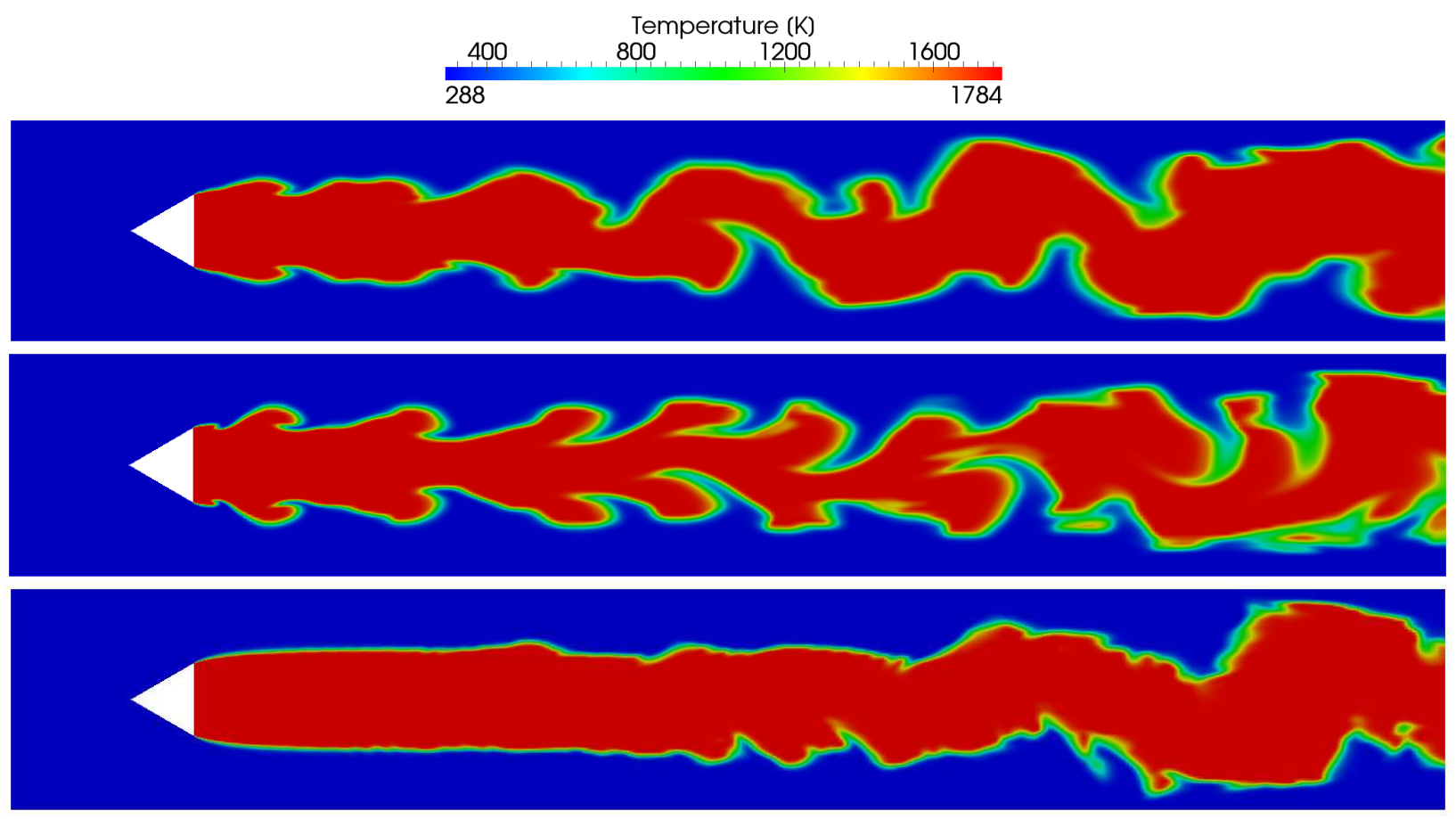

Figure 14: Instantaneous temperature contours showing sinusoidal motion. Top to bottom: CHARLES, LESLIE3D, and Fluent.

With the trend of event arrival rate and event duration both decreasing with increasing density ratio it would be reasonable to assume the appearance of an asymmetric flame under density ratio of 6 conditions, should be extremely rare and possibly non-existent. However, when studying a time series of images from the CHARLES simulation it can be estimated that the flame is asymmetric at least $20 \%$ of the time. This suggests the simulations may be on the verge of going fully asymmetric and small modifications to the modeling or simulation setup could facilitate this transition. This could also explain the presence of many asymmetric solutions in the literature.

One such change in simulation setup which could trigger full asymmetry is a change in the numerical scheme used. Figure 15 shows results from LESLIE3D, and compares the instantaneous temperature contours shown before that were obtained using the MUSCL scheme, with those obtained when using a central scheme, to discretize the governing system of equations. The central scheme predicts a permanently asymmetric flame shape with very little vortex motion in the flame sheet. This demonstrates the ability to predict drastically different solutions with a given physical sub-grid model and given computational grid, even in a single code, when different numerical methods are used and hence a different numerical error is present. The nature of the error, range of scales impacted by the error and the absolute magnitude of the error can be different for different numerical schemes. The interaction of numerical error with physical sub-grid models is therefore code dependent and can result in different solutions. This interaction of errors appears to be much more severe in the simulation of this reacting flow than that reported in the past for the simulation of non-reacting flows ${ }^{6-14,16,17}$, and has the potential to change the fundamental nature of the flow. Flame location and shape has a first-order impact on combustion dynamics and emissions and hence the change from a symmetric to an asymmetric flame shape due to the interaction of numerical and modeling errors significantly impacts the predictive capabilities of the computational approach. A detailed analysis of why the numerical error impacts the predictive capabilities of reacting flow LES, along with research aimed at mitigating its impact, should be the focus of future work by the LES community. 


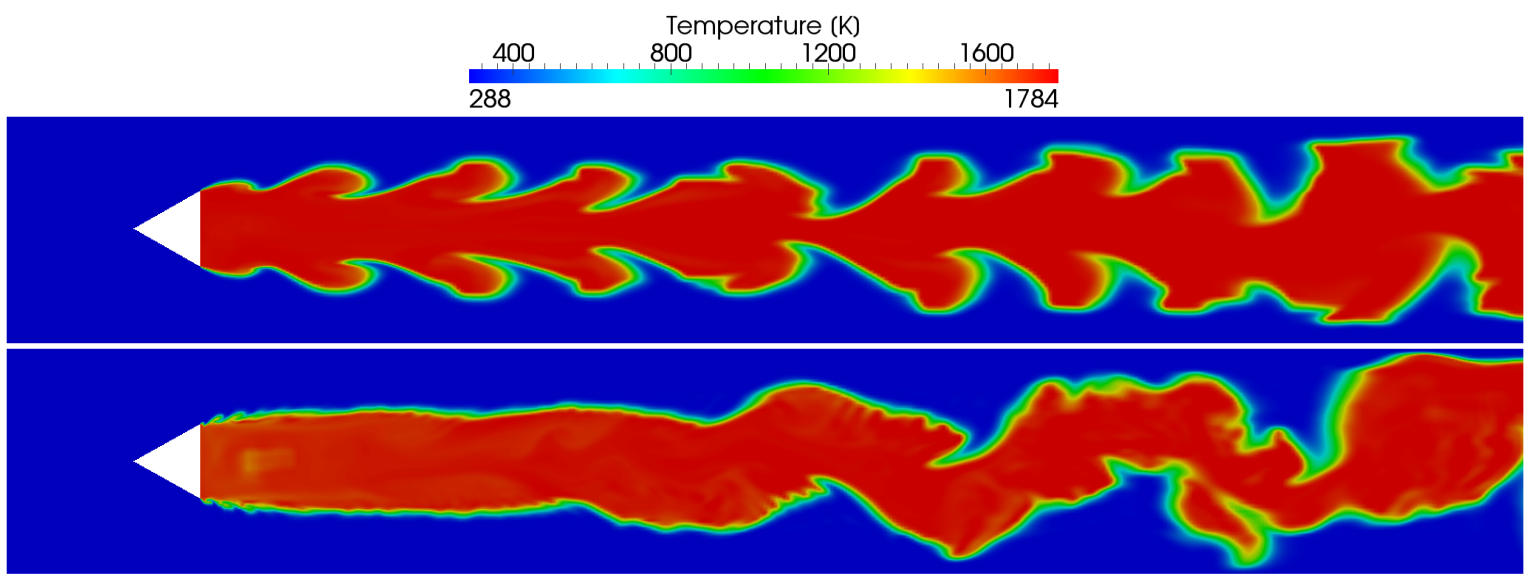

Figure 15: Instantaneous temperature contours from LESLIE3D simulations that utilize a MUSCL scheme (top) and a central scheme (bottom).

Returning to Figure 12, it is noted that even though CHARLES, LESLIE3D and Fluent can be seen to be in qualitative agreement for the flame shape, they do exhibit some differences with respect to flame wrinkling and vortex motion. Differences between computational results and experimental data could be blamed on the physical sub-grid models employed. However, given that the physical sub-grid models and computational grids are the same for each code, such differences between the results from each code must be attributed to the different numerical methods each code employs.

In Figure 12, the Fluent solution shows very little flame wrinkling and hence minimal vortex motion is present, suggesting higher levels of numerical dissipation. However, the regions of high progress variable gradient are sharply captured. Both CHARLES and LESLIE3D capture similar vortex behavior; in the upper half of the combustor duct, the direction of vortex rotation is clockwise just downstream of the bluff body, but switches to an anti-clockwise direction further downstream. The directions of rotation are in the opposite sense for the lower half of the duct. These phenomena are physical ${ }^{29,42,43}$ and are caused by the generation of baroclinic voriticy by the flame.

This switching in direction of rotation is also demonstrated by the mean z-vorticity contours presented in Figure 16, where a switch in the sign of this quantity can clearly be seen in each half of the domain. Studying these mean fields also demonstrates that this is a persistent phenomenon, not simply an occurrence in a particular instantaneous image. It can also be seen from Figure 16 that the levels of mean z-vorticity are weaker in the Fluent solution, as might be expected from the lack of clear vortex motion in the instantaneous contour of Figure 12.

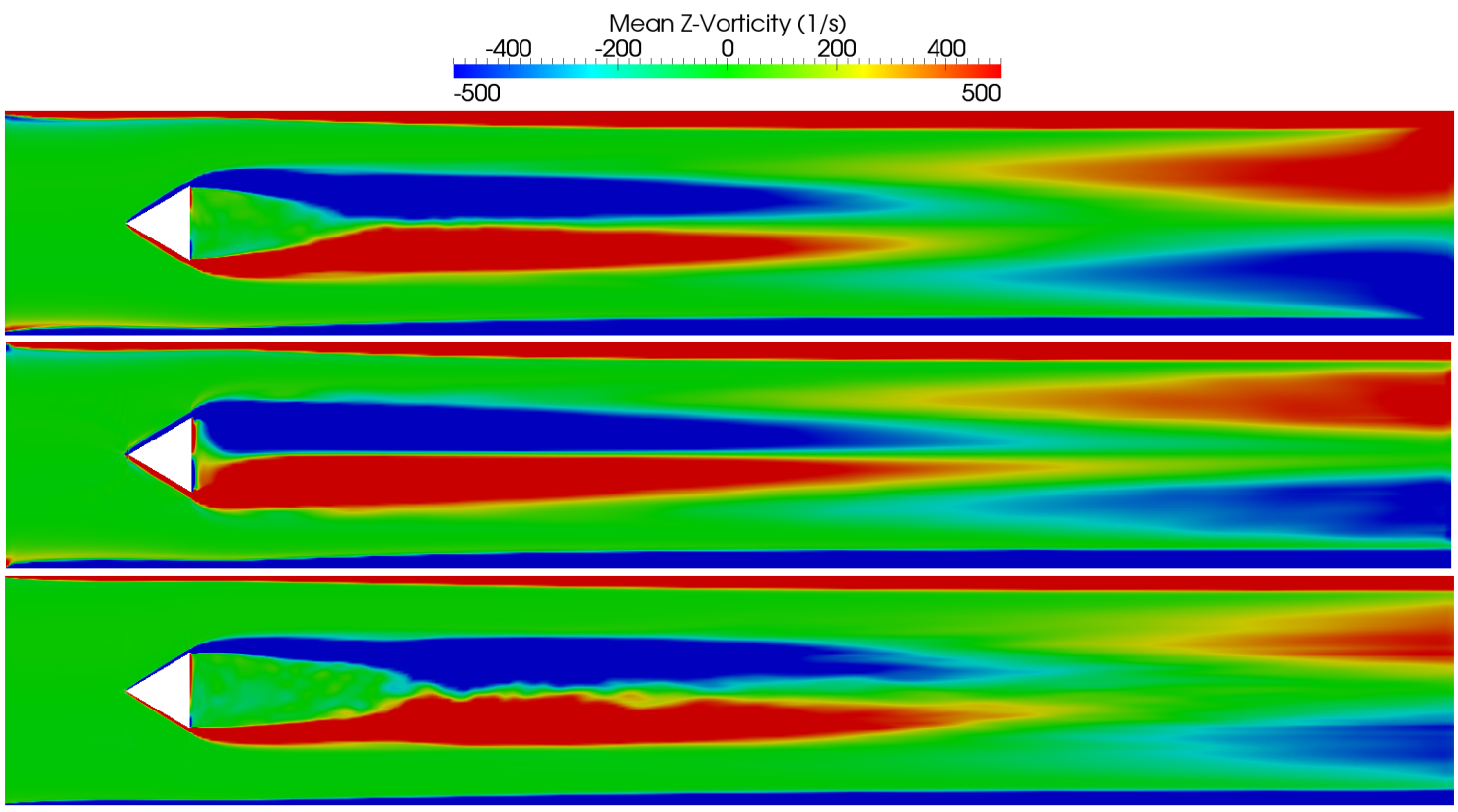

Figure 16: Mean z-vorticity contours. Top to bottom: CHARLES, LESLIE3D, Fluent. 
It can also be seen from Figure 16 that LESLIE3D predicts a different flow field immediately downstream of the bluff body, when compared to CHARLES and Fluent. LESLIE3D predicts the presence of a re-circulation zone, with two regions of circulation with opposite sign, whereas CHARLES and Fluent both predict a dead region behind the bluff body. LESLIE3D is in better agreement with theory ${ }^{44}$ and it will be shown that the presence of such a recirculation zone is also consistent with experimental data. This analysis is supported by Figure 17, where the scale for the z-vorticity contours has been reduced by a factor of ten to ensure a weak re-circulation region is not predicted by CHARLES or Fluent. The mean axial velocity contours behind the bluff body demonstrate the shallow recirculation regions predicted by CHARLES and Fluent. Such behavior is also supported by the instantaneous temperature plots in Figure 12, where LESLIE3D exhibits significantly more rotation in the shear layer immediately downstream of the bluff body, corresponding to the higher levels of z-vorticity in this region.

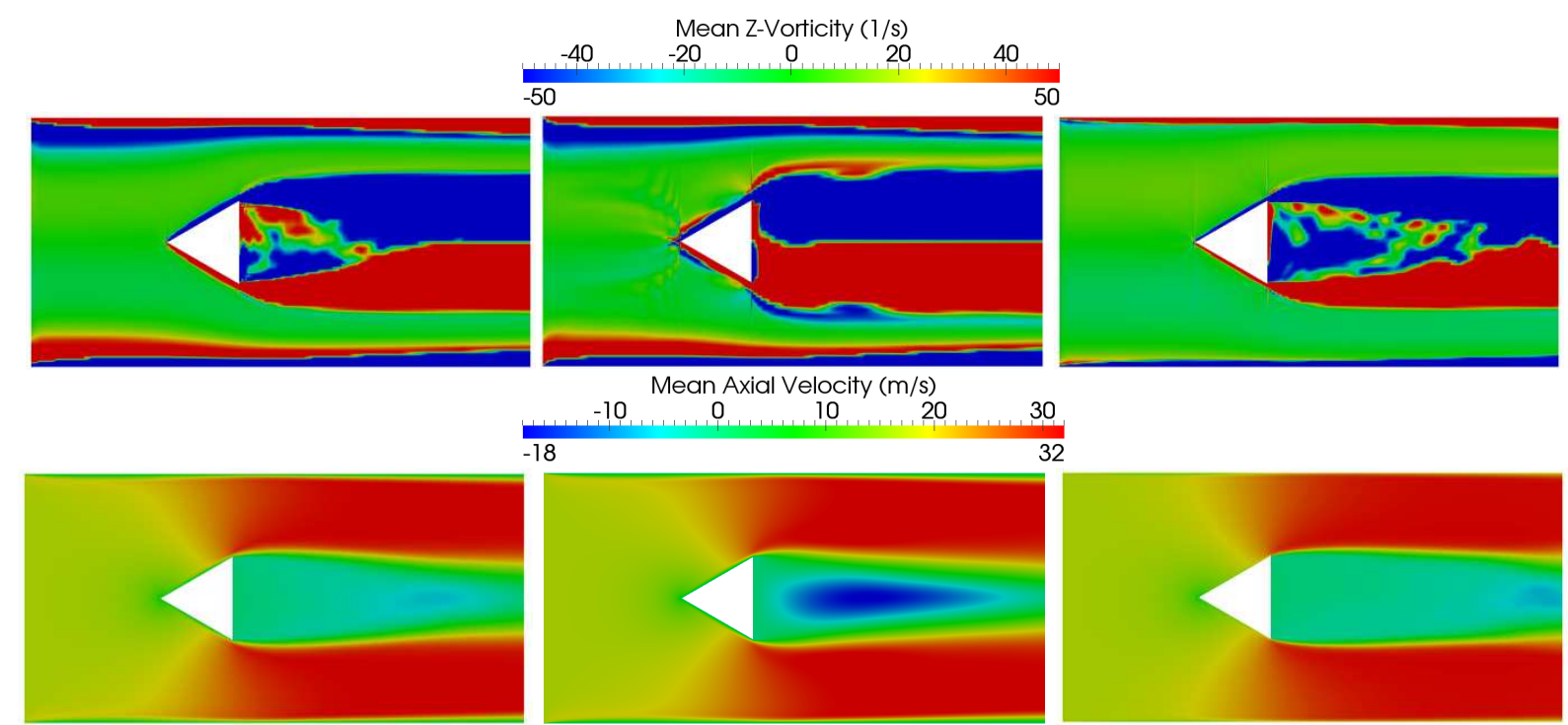

Figure 17: Mean z-vorticity, with scale reduced by a factor of 10 (top), and mean axial velocity (bottom), where scale has been set for the peak velocities from LESLIE3D, in the bluff body region. Left to right: CHARLES, LESLIE3D and Fluent.

CHARLES, LESLIE3D and Fluent have been shown to be in approximate qualitative agreement with respect to flame shape, although some differences in the predicted flow field have already been identified. However, it is important to compare the mean and fluctuating statistics profiles to experimental data in order to investigate the quantitative validity of each solution.

Figure 18 compares centerline profiles for the mean axial velocity, anisotropy and fluctuation level for CHARLES, LESLIE3D and Fluent. Studying the mean axial velocity profile, it is clear that none of the codes are capable of quantitatively re-producing the re-circulation region downstream of the bluff-body. It should be noted that this re-circulation region is important in combustion, since this negative velocity region facilitates flameholding. Hence, its accurate prediction is a first order requirement in reacting simulations. Both CHARLES and Fluent predict a re-circulation region which is too shallow with respect to the velocity magnitude (to the degree that the predicted regions cannot be called 're-circulation' zones), whilst LESLIE3D predicts one which is too deep. This is consistent with the observations made from Figure 16 and Figure 17. However, both CHARLES and LESLIE3D accurately capture the end of the re-circulation region, defined as the location at which the mean axial centerline velocity rises above zero. Although CHARLES and LESLIE3D have difficulty capturing the near-field physics, they are able to accurately predict the centerline velocity profile further downstream. This is quite surprising, since it is reasonable to assume that the near-field solution would impact the results further downstream. It is also interesting to note that, although the re-circulation zone predictions differ between the codes, the slope of the centerline mean axial velocity curves are approximately the same further downstream. This suggests that each code produces the same burning rate. 

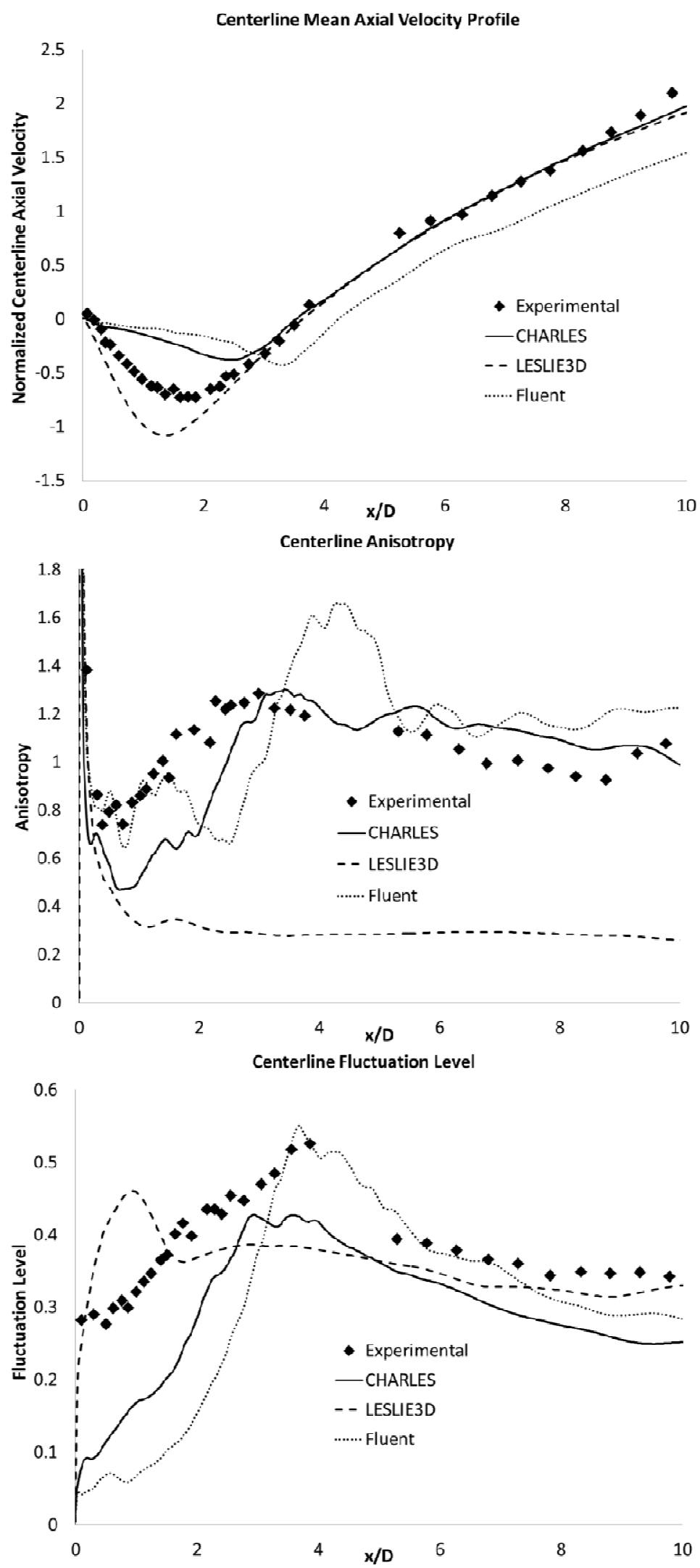

Figure 18: Centerline profiles for the mean axial velocity, anisotropy and fluctuation level on the $2 \mathrm{~mm}$ grid for the reacting solutions from CHARLES, LESLIE3D and Fluent. 
Studying the centerline anisotropy and fluctuation level comparisons in Figure 18, it can be seen that CHARLES and Fluent again provide a poor prediction in the re-circulation region downstream of the bluff body. However, further downstream they provide a reasonable estimation of these centerline quantities. Comparing to Figure 7, it can be seen that there is significant variation in the results obtained from each code for the reacting case, with no observable collapse of the computational data, in contrast to the non-reacting result. The anisotropy levels for LESLIE3D in Figure 18 are in poor agreement with experimental data, but the fluctuation levels appear to be reasonable. This can be explained by analysis of the predicted fluctuating components of velocity, which shows LESLIE3D over-estimates $u^{\prime}$ and under-estimates $v^{\prime}$, compared to experimental data and computational results from other codes. This causes very low values of anisotropy, but each component of fluctuating velocity compensates in the calculation of the fluctuation level, leading to values of a reasonable magnitude for this quantity. It should be noted, however, that LESLIE3D has been shown in other published work to predict this flow-field in close agreement with the experimental data when employing different models for the sub-grid closure.

It could be argued that, although the $2 \mathrm{~mm}$ resolution grid has been demonstrated to be sufficient for the nonreacting flow field, a finer resolution could be required to capture the physics of the reacting case. This is because the sub-grid kinetic energy of a reacting flow is not consistent with the incompressible non-reacting analysis used to estimate the required grid resolution. As a result, a reacting simulation is also conducted on a $1 \mathrm{~mm}$ resolution grid in CHARLES, with the centerline mean axial velocity presented in Figure 19. It can be seen that minimal differences are apparent between the $2 \mathrm{~mm}$ and $1 \mathrm{~mm}$ resolution solutions. Therefore, it is reasonable to assume the $2 \mathrm{~mm}$ resolution grid is also sufficient for the reacting simulations.



Figure 19: Centerline profiles for the mean axial velocity on $2 \mathrm{~mm}$ and $1 \mathrm{~mm}$ grids for the reacting solutions from CHARLES.

Figure 20 demonstrates that even though the statistical field is not altered by a refinement of the mesh, a higher turbulence content, made up of finer scales, is captured. Although these instantaneous contours also appear to show an improved re-circulation zone on the $1 \mathrm{~mm}$ resolution grid, the mean z-vorticity contours in Figure 21 show that this is only an occurrence in the instantaneous image since the dead region is still present behind the bluff body in the mean field. 


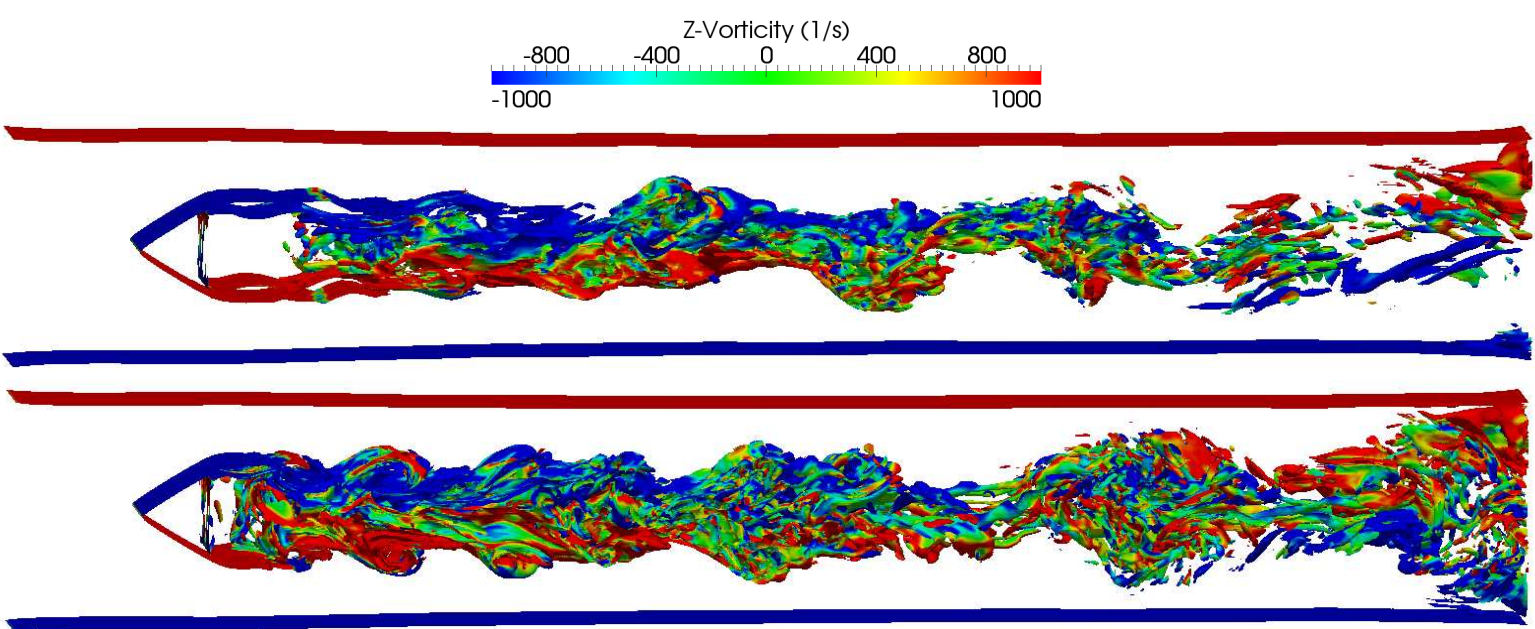

Figure 20: Isosurfaces of vorticity magnitude at level $2000^{\mathrm{s}-1}$ for the reacting simulations from CHARLES, colored by the $Z$ component of vorticity, on the $2 \mathrm{~mm}$ (top) and $1 \mathrm{~mm}$ (bottom) grid resolutions.



Figure 21: Mean z-vorticity contours from CHARLES. Top to bottom: $2 \mathrm{~mm}$ grid and $1 \mathrm{~mm}$ grid.

For completeness, the mean axial velocity and Reynolds stress profiles across the flame are presented in Figure 22 and Figure 23, respectively. As expected, CHARLES and Fluent have comparable mean velocity profiles within the re-circulation zone, but the peak velocity in Fluent is below that of CHARLES, further downstream. Although the depth of the re-circulation zone is more accurately predicted by LESLIE3D at some axial locations, the height of this zone (i.e. y/D width) is poorly captured. With regard to the Reynolds stress profiles, both CHARLES and Fluent start off with very flat profiles, in poor agreement with experimental data, but grow further downstream to be in better agreement at these locations. The results from LESLIE3D are the contrast, where shear layer fluctuations exist close to the bluff body and are in good agreement with experimental measurements. However, further downstream the Reynolds stress profiles for LESLIE3D become flat and are in poor agreement with the experimental results. This is somewhat surprising when studying Figure 12, since LESLIE3D has the largest flame unsteadiness at the large scale implying an associated level of flow unsteadiness.

Mean temperature profiles across the flame are presented in Figure 24. It can be seen that the spreading rate of the flame is under predicted in all simulations. Due to the lack of flame motion just downstream of the bluff body in the Fluent simulation, a very square temperature profile is obtained. Due to the significant flame motion for LESLIE3D, a rounded peak in the temperature profile is evident even at the first axial location. The results from CHARLES are between the two, consistent with the observations of the qualitative results.

It is surprising that the mean velocity profiles are in reasonable agreement with the experimental data, when the spreading rate of the flame is under predicted, since this under prediction would suggest lower heat release compared to the experiment. However, the peak temperatures from the simulations are consistently higher than in the experiment and this may compensate against this effect. The higher computational values could be due to heat 
losses in the experiment and also uncertainties in the experimental equivalence ratio. If the adiabatic flame temperature is lowered in the simulations (i.e. a reduction in the equivalence ratio), or heat losses are taken into account, the downstream acceleration achieved in the computational results may not be as high. The high peak temperatures in the computational results can also be attributed to the lack of mixing in the simulations, at least in the bluff body near field. This can be attributed to the poor prediction of the re-circulation region by all codes. Higher mixing would introduce cooler reactants into the region now occupied by the products and reduce the peak average temperatures observed.
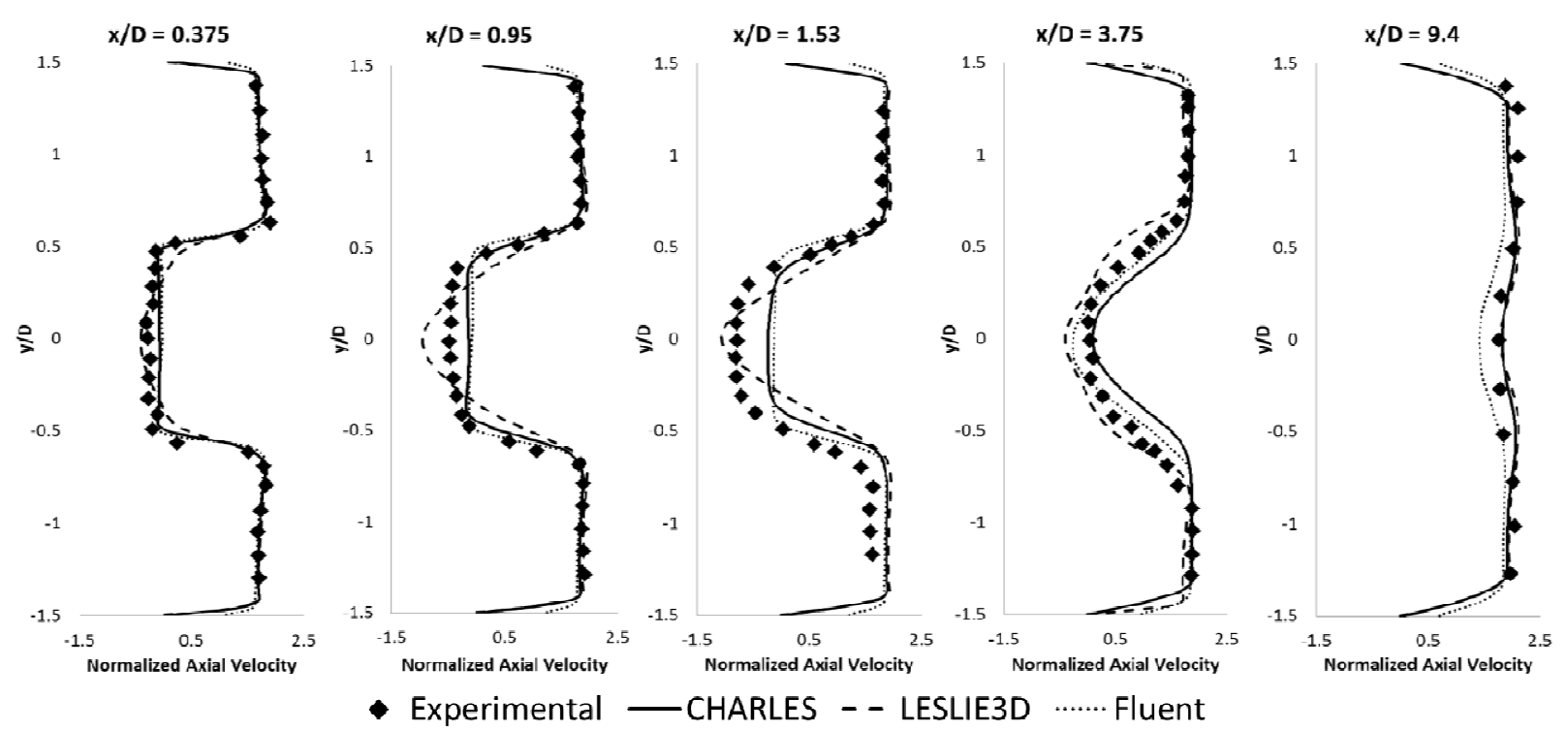

Figure 22: Normalized mean axial velocity profiles across the flame, on the $2 \mathrm{~mm}$ grid, at several axial (x) locations for the reacting case.


Figure 23: Normalized mean Reynolds stress profiles across the flame, on the $2 \mathrm{~mm}$ grid, at several axial (x) locations for the reacting case. 



Figure 24: Mean temperature profiles across the flame, on the $2 \mathrm{~mm}$ grid, at several axial (x) locations for the reacting case. 
Given the results presented in this section it is clear that, unlike the non-reacting case, none of the codes employed have been able to accurately capture the reacting flow field. While all of the codes approximate the experimentally observed behavior to a certain degree, significant discrepancies remain. The discrepancies range from an inability to predict qualitative large scale features such as the presence, or not, of a sinus shedding mode and of a robust recirculation zone behind the bluff body, to quantitative misses in the mean velocity and temperature profiles and second order turbulence statistics. The errors in the latter are most significant both in magnitude and their potential impact given the paramount importance of turbulence to mixing and chemical reaction in reacting flows. The overall inaccuracies in the obtained solutions are large enough for one to suspect that it may be possible that none of these solutions are actually correct and that other physical manifestations of the flow can better match the experimental data. In order to conclusively state this, however, an increase in the fidelity of the experimental data is required so that the actual physical manifestation of the flow can be described with certainty.

In the context of the objectives of this paper, however, the lack of consistency between the results from the various codes, e.g. one code does better in the near field, another in the far field, the instantaneous solutions look qualitatively different etc., is much more significant than their inability to accurately reproduce the experimentally observed flow. If all the codes predicted consistently problematic solutions then the physical sub-grid models and computational grid could be blamed. Rather, the lack of consistency, given a fixed set of sub-grid models and grids, points to issues related to the interaction of the numerical methods used to solve the conservation equations. Mitigation of this impact of numerical error on the solutions obtained is required to improve the predictive capabilities of the LES approach. More importantly, this mitigation must be accomplished prior to concentrating on physical sub-grid model improvements. Development and demonstration of a physical sub-grid model in one code, in the presence of one set of numerical methods and associated numerical error, does not guarantee predictive capabilities or even satisfactory performance when transitioned to another code, which uses different numerical methods to solve the conservation equations.

It is also important to note that it is unlikely that more sophisticated models would do better in this respect. The more sophisticated models would preserve physical non-linearities better and would respond even more strongly to the numerical scheme inaccuracies and enable even more solutions with changes in the numerical parameters.

What is clear from the above discussion is that further research is needed to better understand the coupling of numerical methods with sub-grid models. Fundamental corrections may be needed but, in the minimum, better rules of application are probably required e.g. resolution requirements that in addition to the physics are linked to the numerical scheme and sub-grid model used. This, of course, will also have to deal with the fact that most sub-grid models are inherently grid dependent. Finally, it is recognized that resolution of this issue will only provide consistency of solution. Once this is achieved, research is also required to enhance the physics captured by the subgrid models. These activities will be the focus of future research.

\section{Conclusions}

The bluff-body stabilized combustion experiment of Volvo Flygmotor $\mathrm{AB}$ was simulated to highlight the challenges facing the use of LES as a predictive tool for realistic combustion systems. The focus was on assessing the impact of numerics on the LES solution. Four different LES codes were used, all involving different numerical schemes but the same physical sub-grid models and computational grids. Reacting and non-reacting solutions were obtained. The main findings and conclusions are as follows:

1. All codes produced similar non-reacting solutions which were accurate approximations of the experimentally observed flow field in terms of dynamics, mean profiles and second order turbulence statistics. At the coarsest resolution, one of the codes transitioned to a different flow solution. It was concluded that the impact of numerical error near solution transition regions can be significant.

2. The codes produced solutions for the reacting flow which were inconsistent among themselves and which faced challenges when compared to the experimentally observed flow:

- Only three out of four codes were able to capture the suppression of asymmetric vortex shedding and the prediction of a predominantly symmetric flame

- The three codes that produced predominantly symmetric solutions showed signs of being too close to the transition to asymmetric behavior; they experienced frequent asymmetric shedding events and in one code a change in the numerical scheme lead to full asymmetry.

- The three codes with predominantly symmetric solutions correctly predicted a change in sign in the downstream z-vorticity, but to differing degrees, i.e. they were not in quantitative agreement. 
- These codes were unable to quantitatively predict the re-circulation zone details, a region of significant importance in a reacting flow. Only one code predicted a robust recirculation zone, the other two predicted effectively stagnant regions.

- Mean velocity and temperature profiles were approximately predicted but much less accurately than the non-reacting case.

- Second order statistics were poorly predicted, with a high degree of variation between the codes

- The spreading rate of the flame was under-predicted

3. Key Conclusion I: The shortcomings in the reacting solutions that are consistent between the codes may be blamed on the sub-grid models chosen. The inconsistencies between these solutions while using the same physical sub-grid models and computational grids, on the other hand, must be linked to the interaction of the numerical scheme with the sub-grid models and not the sophistication of the models themselves.

4. Key conclusion II: Significant advances in the understanding of the mechanisms by which numerical error impacts reacting LES are required, that lead to new and computationally affordable methodologies for the mitigation of this impact. Removing the grid dependency of sub-grid models is probably a first step in this direction $^{13}$. So are techniques that can provide insight into the sources of numerical error, such as explicit filtering ${ }^{8-13}$.

5. Additional LES set up metrics and criteria are required for reacting flows, since those developed for incompressible non-reacting flow are likely not adequate. To achieve this, advances in the fundamental understanding of the impact of combustion on turbulence are required - e.g. in order to help arrive at better estimates of sub-grid kinetic energy levels.

6. Effective validation of reacting LES requires experimental data that should meet special requirements:

- Experimental cases should have significant combustion energy release so that flow is not dominated by cold flow features

- Time depended flow features need to be quantified (e.g. time accurate flow visualization). Turbulence statistics are not always sufficient.

- High fidelity measurements need to be made with error quantification so that approximate but incorrect solutions may be excluded.

\section{Acknowledgments}

The authors would like to thank Cascade Technologies Inc. and Prof. Suresh Menon's group for access to their respective software and for useful technical discussions. Computational support for some of this work from the Oak Ridge Leadership Computing Facility at Oak Ridge National Laboratory, which is supported by the Office of Science of the Department of Energy under Contract DE-AC05-00OR2272, is also gratefully acknowledged. This work was supported by UTRC internal resources.

\section{References}

${ }^{1}$ Pope, S.B., "Turbulent Flows", Cambridge University Press, 2000

${ }^{2}$ Vreman, B., Geurts, B. and Kuerten, H., "Comparison of Numerical Schemes in Large-Eddy Simulation of the Temporal Mixing Layer", Intl. J. Numer. Meth. Fluids, 1996, 22, pp. 297-311

${ }^{3}$ http://www.sandia.gov/TNF/

${ }^{4}$ Moureau, V., Domingo, P. and Vervisch, L., "From Large-Eddy Simulation to Direct Numerical Simulation of a lean premixed swirl flame: Filtered laminar flame-PDF modeling", Combustion and Flame, 158, 7, 2011, pp. 13401357

${ }^{5}$ Tucker, P. K., Menon, S., Merkle, C. L., Oefelein, J. C. and Yang, V., "Validation of High-Fidelity CFD Simulations for Rocket Injector Design", 44 $4^{\text {th }}$ AIAA/ASME/SAE/ASEE Join Propulsion Conference \& Exhibit, 2008, AIAA-2008-5226

${ }^{6}$ Ghosal, S. and Moin, P., "The Basic Equations for the Large Eddy Simulation of Turbulent Flows in Complex Geometry", J. Comp. Phys., 118, pp.24-37, 1995

${ }^{7}$ Ghosal, S. An analysis of numerical errors in large-eddy simulations of turbulence. J. Comp. Phys. 125, 187206. 
${ }^{8}$ Lund, T. S., "The Use of Explicit Filters in Large Eddy Simulation", Computers and Mathematics with Applications, 46, 2003, pp. 603-616

${ }^{9}$ Radhakrishnan, S. and Bellan, J., "Explicit filtering to obtain grid-spacing-independent and discretization-orderindependent large-eddy simulation of compressible single-phase flow", J. Fluid Mech., 2012, 697, pp. 399-435

${ }^{10}$ Bose, S. T. and Moin, P. and You, D., "Grid-independent large eddy simulation using explicit filtering", Physics of Fluids, 22, 105103, 2010

${ }^{11}$ Gullbrand, J. and Chow, F. K., "The effect of numerical errors and turbulence models in large-eddy simulations of channel flow, with and without explicit filtering", J. Fluid Mech., 2003, 495, pp. 323-341

${ }^{12}$ Mathew, J., Lechner, R., Foysi, H., Sesterhenn, J. and Friedrich, R., "An explicit filtering method for large eddy simulation of compressible flows", Physics of Fluids, 15, 8, 2003, pp.2279-2289

${ }^{13}$ Winckelmans, G. S., Wray, A. A., Vasilyev, O. V. and Jeanmart, H., "Explicit-filtering of large-eddy simulation using the tensor-diffusivity model supplemented by a dynamic Smagorinsky term", Physics of Fluids, 13, 5, 2001, pp.1385-1403

${ }^{14}$ Meyers, J., Geurts, B., and Sagaut, P., "Quality and Reliability of Large Eddy Simulations”, Springer, 2008

${ }^{15}$ Cocks, P. A. T, Sankaran, V. and Soteriou, M. C., "Towards Predictive Reacting Flow LES", AIAA $52^{\text {nd }}$ Aerospace Sciences Meeting, 2014, AIAA 2014-0826

${ }^{16}$ Klein, M., Meyers, J., Geurts, B. J., Assessment of LES quality measures using the error landscape approach, "Quality and Reliability of Large Eddy Simulations", Springer, pp.131-142, 2008

${ }^{17}$ Kempf, A. M., Geurts, B. J., and Oefelein, J. C., "Error analysis of large eddy simulation of the turbulent nonpremixed Sydney bluff-body flame", Combustion and Flame, 158, 12, 2011, pp. 2408-2419

${ }^{18}$ Sjunnesson A., Olovsson S. and Sjöblom B., "Validation Rig - A Tool for Flame Studies", Technical Report 9370-308, 1991

${ }^{19}$ Sjunnesson A., Nelsson C and Max E., "LDA Measurements of Velocities and Turbulence in a Bluff Body Stabilized Flame", Fourth International Conference on Laser Anemometry - Advances and Application, ASME, Cleveland, August 1991

${ }^{20}$ Sjunnesson, A., Henrikson, P. and Lofstrom, C., "CARS Measurements and visualization of reacting flows in a bluff body stabilized flame", $28^{\text {th }}$ Joint Propulsion Conference and Exhibit, Nashville, TN, July 1992, AIAA 923650

${ }^{21}$ Clavin, P. and Williams, F. A., "Theory of premixed-flame propagation in large-scale turbulence", J. Fluid Mech., 1979, 90, 3, pp. 589-604

${ }^{22}$ Fureby, C., "A fractal flame-wrinkling large eddy simulation model for premixed turbulent combustion", Proceedings of the combustion Institute, 30, 2005, pp. 593-601

${ }^{23}$ Fureby, C., "A computational study of combustion instabilities due to vortex shedding", Proceedings of the combustion Institute, 28, 2000, pp. 783-791

${ }^{24}$ Porumbel, I. and Menon, S., "Large Eddy Simulation of bluff-body stabilized premixed flames", $44^{\text {th }}$ AIAA Aerospace Sciences Meeting, 2006, AIAA 2006-0152 (2006)

${ }^{25}$ Giacomazzi, E., Battaglia, V. and Bruno, C., "The coupling of turbulence and chemistry in a premixed bluffbody flame as studied by LES", Combustion and Flame, 138, 2004, pp. 320-335

${ }^{26}$ Nilsson, P. and Bai, X. S., "Level-set flamelet library approach for premixed turbulent combustion", Experimental Thermal and Fluid Science, 21, 2000, pp. 87-98

${ }^{27}$ Baudoin, E., Yu, R., Nogenmyr, K. J., Bai, X. S. and Fureby, C., "Comparison of LES Models Applied to a Bluff- Body Stabilized Flame", $47^{\text {th }}$ Aerospace Sciences Meeting, 2009, AIAA 2009-1178

${ }^{28}$ Martensson, H., "Numerical Simulations of Unsteady WakeFlow", International Society for Air-breathing Engines Conference, 1991, ISABE-91-7104

${ }^{29}$ Erickson, R. R. and Soteriou, M. C., "The influence of reactant temperature on the dynamics of bluff body stabilized premixed flames", Combustion and Flame, 158, 2011, pp. 2441-2457

${ }^{30}$ Akula, R. K., Sadiki, A., Janicka, J. and Warnatz, J., "Evaluation of Large Eddy Simulation of Premixed Turbulent Combustion Using Flame Surface Density Approach", Third European Combustion Meeting ECM-2007

${ }^{31}$ Manickam, B., Franke, J., Muppala, S. P. R. and Dinkelacker, F., "LES of Triangular-stabilized Lean Premixed Turbulent Flames with an algebraic reaction closure: Quality and Error Assessment", Quality and Reliability of Large-Eddy Simulations II, ERCOFTAC Series, 16, 2011, pp. 221-230

${ }^{32}$ Gokulakrishnan, P., Foli, K., Klassen, M., Roby, R., Soteriou, M., Kiel, B. and Sekar, B., "LES-PDF Modeling of Flame Instability and Blow-out in Bluff-Body Stabilized Flames", $45^{\text {th }}$ AIAA/ASME/SAE/ASEE Joint Propulsion Conference, August 2009, Denver, CO, AIAA-2009-5409 
${ }^{33}$ Manickam, B., Franke, J., Muppala, S. P. R. and Dinkelacker, F., "Large-eddy Simulation of Triangularstabilized Lean Premixed Turbulent Flames: Quality and Error Assessment", Flow Turbulence Combustion, 88, 2012, pp. 563-596

${ }^{34}$ Tuttle, S. G., Chaudhuri, S., Kostka Jr., S., Kopp-Vaughan, K. M., Jensen, T. R., Cetegen, B. M. and Renfro, M. W., "Time-resolved blowoff transition measurements for two-dimensional bluff body-stabilized flames in vitiated flow", Combustion and Flame, 159, 2012, pp. 291-305

${ }^{35}$ Huelskamp, B. C., Kiel, B. V., Lynch, A. C., Kostka, S., Gokulakrishnana, P. and Klassen, M. S., "Improved Correlation for Blowout of Bluff-Body Stabilized Flames", $49^{\text {th }}$ AIAA Aerospace Sciences Meeting, Orlando, FL, January 2011, AIAA-2011-66

${ }^{36}$ Emerson, B., O’Connor, J., Juniper, M. and Lieuwen, T., "Density ratio effects on reacting bluff-body flow field characteristics", J. Fluid. Mech, 2012, 706, pp. 219-259

${ }^{37}$ Smagorinsky, J., "General Circulation Experiments with the Primitive Equations. I. The Basic Experiment", Monthly Weather Review, 91, 1963, pp.99-164

${ }^{38}$ Gottlieb, S. and Shu, C.-W., "Total Variation Diminishing Runge-Kutta Schemes", Mathematics of Computation, 67, 221, 1998, pp.73-85

${ }^{39}$ Weller, H. G., Tabor, G., Gosman, A. D., and Fureby, C., “Application of a flame-wrinkling LES combustion model to a turbulent mixing layer”, Proceedings of the combustion Institute, 27, 1998, pp. 899-907

${ }^{40}$ Schlichting, H., "Boundary Layer Theory", McGraw-Hill, 1955

${ }^{41}$ Cocks, P. A. T., Sankaran, V. and Soteriou, M. C., "Is LES of reacting flows predictive? Part 1: Impact of numerics", AIAA 51 ${ }^{\text {st }}$ Aerospace Sciences Meeting, 2013, AIAA 2013-0170

${ }^{42}$ Mehta, P. G. and Soteriou, M. C., "Combustion Heat Release Effects On The Dynamics Of Bluff Body Stabilized Premixed Reacting Flows", 41 $1^{\text {st }}$ Aerospace Sciences Meeting and Exhibit, January 2003, Reno, NV, AIAA-2003-0835

${ }^{43}$ Shanbhogue, S. J., Husain, S. and Lieuwen, T., "Lean blowoff of bluff body stabilized flame: Scaling and dynamics", Progress in Energy and Combustion Science, 35, 2009, pp.98-120

${ }^{44}$ Glassman, I., "Combustion", Academic Press, 1987 
Figure 1: Comparison of methods used for thermodynamic coupling

Figure 2: Computational grid (4mm mesh) showing clustering near walls and isotropic mesh away from walls

Figure 3: Isosurfaces of vorticity magnitude at levels $2000 \mathrm{~s}^{-1}$ and $4000 \mathrm{~s}^{-1}$ for a non-reacting simulation from CHARLES on the $2 \mathrm{~mm}$ resolution grid, colored by the $Z$ component of vorticity.

Figure 4: Centerline profiles for the mean axial velocity, anisotropy and fluctuation level on the $4 \mathrm{~mm}$ resolution grid for the non-reacting case.

Figure 5: Comparison of mean axial velocity contours from CHARLES (top) and LESLIE3D (bottom) on the $4 \mathrm{~mm}$ resolution grid, for the non-reacting case.

Figure 6: Comparison of instantaneous z-vorticity contours from CHARLES (top) and LESLIE3D (bottom) on the $4 \mathrm{~mm}$ resolution grid, for the non-reacting case.

Figure 7: Centerline profiles for the mean axial velocity, anisotropy and fluctuation level on the $2 \mathrm{~mm}$ resolution grid for the non-reacting case.

Figure 8: Normalized mean axial velocity wake profiles on the $2 \mathrm{~mm}$ grid, at several axial (x) locations for the non-reacting case.

Figure 9: Normalized mean Reynolds stress wake profiles on the $2 \mathrm{~mm}$ grid, at several axial $(x)$ locations for the non-reacting case.

Figure 10: Instantaneous contours of percent of resolved kinetic energy, on both the $4 \mathrm{~mm}$ and $2 \mathrm{~mm}$ resolution grids, for the non-reacting simulations from (top to bottom): CHARLES, LESLIE3D, OpenFOAM and Fluent.

Figure 11: Isosurfaces of vorticity magnitude at level $2000 \mathrm{~s}^{-1}$ for the reacting simulation from CHARLES, colored by the $Z$ component of vorticity.

Figure 12: Instantaneous temperature contours. Top to bottom: CHARLES, LESLIE3D, OpenFOAM and Fluent.

Figure 13: Mean centerline axial velocity profile on the $2 \mathrm{~mm}$ grid for the reacting solution from OpenFOAM.

Figure 14: Instantaneous temperature contours showing sinusoidal motion. Top to bottom: CHARLES, LESLIE3D, and Fluent.

Figure 15: Instantaneous temperature contours from LESLIE3D simulations that utilize a MUSCL scheme (top) and a central scheme (bottom).

Figure 16: Mean z-vorticity contours. Top to bottom: CHARLES, LESLIE3D, Fluent.

Figure 17: Mean z-vorticity, with scale reduced by a factor of 10 (top), and mean axial velocity (bottom), where scale has been set for the peak velocities from LESLIE3D, in the bluff body region. Left to right: CHARLES, LESLIE3D and Fluent.

Figure 18: Centerline profiles for the mean axial velocity, anisotropy and fluctuation level on the $2 \mathrm{~mm}$ grid for the reacting solutions from CHARLES, LESLIE3D and Fluent.

Figure 19: Centerline profiles for the mean axial velocity on $2 \mathrm{~mm}$ and $1 \mathrm{~mm}$ grids for the reacting solutions from CHARLES. 
Figure 20: Isosurfaces of vorticity magnitude at level $2000^{\mathrm{s}-1}$ for the reacting simulations from CHARLES, colored by the $Z$ component of vorticity, on the $2 \mathrm{~mm}$ (top) and $1 \mathrm{~mm}$ (bottom) grid resolutions.

Figure 21: Mean z-vorticity contours from CHARLES. Top to bottom: $2 \mathrm{~mm}$ grid and 1mm grid.

Figure 22: Normalized mean axial velocity profiles across the flame, on the $2 \mathrm{~mm}$ grid, at several axial (x) locations for the reacting case.

Figure 23: Normalized mean Reynolds stress profiles across the flame, on the $2 \mathrm{~mm}$ grid, at several axial (x) locations for the reacting case.

Figure 24: Mean temperature profiles across the flame, on the $2 \mathrm{~mm}$ grid, at several axial (x) locations for the reacting case.

Table 1: Time steps used with each code 